\title{
On-the-fly Symmetrical Quasi-classical Dynamics with Meyer-Miller Mapping Hamiltonian for the Treatment of Nonadiabatic Dynamics at Conical Intersections
}

Deping $H u^{\dagger t^{\circ}}, Y u X i e^{\dagger t^{\circ}}$, Jiawei Peng ${ }^{\dagger+t}$, Zhenggang Lan ${ }^{\dagger *}$

${ }^{\dagger}$ SCNU Environmental Research Institute, Guangdong Provincial Key Laboratory of Chemical Pollution and Environmental Safety \& MOE Key Laboratory of Environmental Theoretical Chemistry, South China Normal University, Guangzhou 510006, China

${ }^{t}$ School of Environment, South China Normal University, Guangzhou 510006, China

KEYWORDS: Meyer-Miller model, on the fly, quasi-classical dynamics, nonadiabatic dynamics, conical intersection 


\section{Abstract}

The on-the-fly version of the symmetrical quasi-classical dynamics method based on the MeyerMiller mapping Hamiltonian (SQC/MM) is implemented to study the nonadiabatic dynamics at conical intersections of polyatomic systems. The current on-the-fly implementation of the SQC/MM method is based on the adiabatic representation and the dressed momentum. To include the zero-point energy (ZPE) correction of the electronic mapping variables, we employ both the $\gamma$ adjusted and $\gamma$-fixed approaches. Nonadiabatic dynamics of the methaniminium cation $\left(\mathrm{CH}_{2} \mathrm{NH}_{2}{ }^{+}\right)$ and azomethane are simulated using the on-the-fly SQC/MM method. For $\mathrm{CH}_{2} \mathrm{NH}_{2}{ }^{+}$, both two ZPE correction approaches give reasonable and consistent results. However, for azomethane, the $\gamma$-adjusted version of the SQC/MM dynamics behaves much better than the $\gamma$-fixed version. The further analysis indicates that it is always recommended to use the $\gamma$-adjusted SQC/MM dynamics in the on-the-fly simulation of photoinduced dynamics of polyatomic systems, particularly when the excited-state is well separated from the ground state in the Franck-Condon region. This work indicates that the on-the-fly SQC/MM method is a powerful simulation protocol to deal with the nonadiabatic dynamics of realistic polyatomic systems. 


\section{Introduction}

The theoretical description of the nonadiabatic dynamics at conical intersections (CIs) in complex systems always arouses great research interests due to the breaking down of the BornOppenheimer (BO) approximation and the involvement of a large number of degrees of freedom (DoFs). ${ }^{1-3}$ Various theoretical approaches were developed to simulate the nonadiabatic dynamics, ${ }^{1-}$

${ }^{10}$ which for example include the full quantum dynamics ${ }^{2,11-15}$ and different versions of mixed quantum-classical/semi-classical dynamics. ${ }^{1-2,5-6,8-10,16-26}$ The complex potential energy functions of the polyatomic molecules with many DoFs impose some additional challenging in the simulation of the nonadiabatic dynamics. To solve such problems, the combination of on-the-fly simulation with various dynamics methods becomes popular in the simulation of the real-time nonadiabatic dynamics of polyatomic systems at all atomic level. ${ }^{5-6,9-10,21,27}$

Because the electronic structure calculations are generally performed at each nuclear time step in the on-the-fly dynamics, the total cost of on-the-fly simulation is rather high. For this reason, on-the-fly simulation of the nonadiabatic dynamics should normally employ practical dynamical methods that can provide a good balance between computational cost and efficiency. Thus, only a few dynamic approaches are possible choices. For example, trajectory surface hopping (TSH) approaches, such as the Tully's fewest switch surface hopping (FSSH) approach and its extension, ${ }^{18,20}$ Landau-Zener/Zhu-Nakamura surface hopping approaches, ${ }^{24,28}$ were employed in the on-the-fly simulation due to their computational efficiency. In recent years, great progress was made to use Tully's on-the-fly FSSH (and its variations) dynamics to treat the nonadiabatic 
dynamics of realistic polyatomic systems. ${ }^{2,9,21,27}$ However, the improper treatment of electronic coherence and frustrated hops in Tully's FSSH approach were widely discussed. ${ }^{8,19-20,29}$ Recent work also tried to combine a more rigorous surface hopping approach ${ }^{30}$ in on-the-fly simulation, which is derived from the exact factorization of the electronic-nuclear wavefunction. ${ }^{31}$ Considerable efforts were made to combine the on-the-fly simulation with the Gaussianwavepacket based and relevant approaches, ${ }^{5,10,32-33}$ such as the ab initio multiple spawning (AIMS), ${ }^{5,10}$ variational multiconfigurational Gaussians (vMCG) ${ }^{32}$ and multiconfigurational Ehrenfest. ${ }^{34}$ Some numerical details should be concerned in the implementation of these approaches. For instance, the new Gaussian wave-packets ${ }^{10}$ should be properly generated in the AIMS and the reasonable basis-function sampling/cloning should be considered in the multiconfigurational Ehrenfest dynamics. ${ }^{35}$ These approaches are very promising because these theoretical frameworks were more rigorous and the computational cost is reasonable.

As expected, only the dynamical approaches with reasonable computational cost may be suitable for on-the-fly dynamics. Particularly, the methods based on independent trajectories, instead of entangled trajectories, are preferred in the implementation of on-the-fly simulation, if both require a similar number of trajectories. According to this idea, it is clear that the symmetrical quasi-classical dynamics based on the Meyer-Miller mapping Hamiltonian (SQC/MM) ${ }^{36-38}$ may be an alternative and suitable approach for on-the-fly simulation. In the Meyer-Miller (MM) mapping model, ${ }^{36}$ a Hamiltonian with $\mathrm{N}$ discrete quantum states is mapped to an effective Hamiltonian (or a mapping Hamiltonian) with $\mathrm{N}$ coupled harmonic oscillators. ${ }^{8,36,39-40}$ It is possible 
to combine the MM mapping Hamiltonian with different dynamics approaches, such as various quasi-classical/semi-classical dynamics approaches ${ }^{41-49}$ quantum-classical Liouville equation (QCLE), ${ }^{50-53}$ path integral and extension, ${ }^{54-59}$ surface hopping, ${ }^{60-61}$ centroid molecular dynamics (CMD) ${ }^{62}$ and ring-polymer molecular dynamics (RPMD) ${ }^{63-69}$ In the quasi-classical dynamics, the inclusion of the zero-point energy (ZPE) in the electronic mapping variables in principle provides better dynamical results than the Ehrenfest dynamics, ${ }^{36}$ while the partial instead of full ZPE should be included in practice. ${ }^{8,70-72}$ When the "bin" technique is taken to perform the initial sampling and the final assignment of the quantum states, the $\mathrm{SQC} / \mathrm{MM}^{38,73}$ is formulated.

The SQC/MM method gained significant attention due to its numerical simplicity and physical insight. ${ }^{38,73-90}$ The reasonable performance of the SQC/MM dynamics was examined by different benchmark works. ${ }^{47,81-82,86-87,89}$ Within the framework of the SQC/MM dynamics, Cotton and Miller suggested different useful approaches in the implementation, such as the triangle windowing technique ${ }^{80,91}$ and the employment of the trajectory-adjusted electronic ZPE correction $^{92}$ in the SQC/MM approach. The SQC/MM method was employed to treat different types of nonadiabatic dynamics, ${ }^{38,73,81-86,93}$ for example excited-state energy/electronic transfer dynamics, $^{73,81,86,90,93}$ singlet fission ${ }^{83-85}$ and scattering dynamics ${ }^{82}$ and photo-dissociation dynamics. ${ }^{92}$ As shown by several benchmark works, ${ }^{47,81-82,86-87}$ the SQC/MM dynamics gives a proper correct description of the nonadiabatic dynamics of various model systems (such as the spin-boson model, etc.), when the bath motion behaves classically. Previous work also proved that the SQC/MM dynamics in principle satisfied the detailed balance in the asymptotic limit. ${ }^{74}$ These 
studies provide us the baseline of the reasonable behaviors of the SQC/MM dynamics. In addition, it is also shown that the electronic coherence of exciton dynamics in photoharvesting and organic photovoltaics systems is captured by the SQC/MM dynamics. ${ }^{81,86}$ Overall, the SQC/MM method is very promising, thus it is highly interesting to introduce it into on-the-fly dynamics for all-atomic simulation of the nonadiabatic dynamics of polyatomic molecules in real time.

Recently, Huo and coworkers proposed to employ the quasi-diabatization procedure in the dynamics propagation. ${ }^{88,94-97}$ They used the quasi-diabatic basis to propagate the MM mapping Hamiltonian based dynamics such as partial-linearized density matrix (PLDM) path-integral approach and SQC/MM approach. Particularly, they made the initial efforts to combine the PLDM path-integral approach, the classical path approximation, and density functional tight-binding (DFTB) calculations to achieve the on-the-fly simulation of nonadiabatic dynamics of photoinduced charge transfer in organic photovoltaic systems. ${ }^{95}$ Late, they extended their approach to study the photoinduced dynamics at CIs. ${ }^{97}$ The quasi-diabatic approach works quite well and improves numerical stability in several cases. When the electronic states involved in the nonadiabatic dynamics have non-neglectable couplings with the higher electronic states, the curl condition is not satisfied. ${ }^{1,98-101}$ In this case, it is not trivial to construct the quasi-diabatic electronic basis within the subspace spanned by a few electronic states unless a much larger number of electronic states are considered in the adiabatic-to-diabatic transformation. The inclusion of many electronic states may bring some additional challenging in the high-level electronic-structure calculations. Alternatively, it is also possible to formulate the MM mapping Hamiltonian in the 
adiabatic representation, ${ }^{36,56,75,102-104}$ providing a natural interface to combine the electronic structure calculations. Along this road, Miller and coworkers ${ }^{75}$ recently re-formulated the SQC/MM dynamics in the adiabatic representation by using the dressed momentum, namely kinematic momentum. This formulism provides us an important starting point for the implementation of on-the-fly SQC/MM dynamics. We noticed that Cui and coworkers tried to combine this approach with the on-the-fly simulation using the semi-empirical electronic-structure calculations. ${ }^{105}$ This work also showed that the on-the-fly SQC/MM dynamics results are sensitive with the choice of the window width and ZPE correction parameter $\gamma$ when $\gamma$ is fixed in the SQC/MM dynamics. In fact, the possible influence of the window width on the dynamics was also discussed in several previous works. ${ }^{77,80}$ This problem may be partially remedied by the trajectoryadjusted electronic ZPE approach proposed by Cotton and Miller. ${ }^{92}$ Thus, in principle, this $\gamma$ adjusted approach is highly recommended in the on-the-fly SQC/MM dynamics to study the photochemistry of realistic systems.

In this work, we try to combine the SQC/MM method with the on-the-fly dynamics. To know more about the performance of the SQC/MM method in on-the-fly nonadiabatic dynamics compared with other methods, such as FSSH and AIMS, two commonly used molecular systems, methaniminium cation $\left(\mathrm{CH}_{2} \mathrm{NH}_{2}^{+}\right)$and azomethane, were chosen in this work. Since we noticed that the choice of the ZPE correction in the SQC/MM dynamics is not a trivial task, we implemented both the $\gamma$-fixed and $\gamma$-adjusted approaches. In the latter case, the $\gamma$ is determined by the initial condition of the electronic DoF for each trajectory. This combination provides a novel 
simulation tool to describe the full-dimensional nonadiabatic dynamics at CIs of polyatomic systems. All implementation is performed based on the nonadiabatic dynamics simulation package

JADE developed in our group. ${ }^{106-107}$ In this sense, we also largely extend the JADE package to allow the on-the-fly dynamics simulation of nonadiabatic dynamics at CIs with various dynamics approaches.

\section{Theory and Methods}

\subsection{SQC/MM Hamiltonian on the adiabatic representation}

The adiabatic representation of MM Hamiltonian can be written as ${ }^{75}$

$$
H=\frac{1}{2 \mathbf{M}} \mathbf{P}_{\text {kin }}^{2}+\sum_{i}^{F}\left(\frac{1}{2} x_{i}^{2}+\frac{1}{2} p_{i}^{2}-\gamma\right) E_{i}(\mathbf{R})
$$

with the kinematic momentum $\mathbf{P}_{\text {kin }}$ defined as

$$
\mathbf{P}_{\text {kin }}=\mathbf{P}+\sum_{i \neq j} x_{i} p_{j} \mathbf{d}_{i j}(\mathbf{R})
$$

where $(\mathbf{R}, \mathbf{P})$ are the coordinates and momenta of the nuclear DoFs, $F$ is the number of electronic states, $\left(x_{i}, p_{i}\right)$ is the coordinate and momentum of the harmonic oscillator that maps to the $i$-th electronic state, $E_{i}(\mathbf{R})$ denotes the energy of the $i$-th electronic state, $\gamma$ represents the ZPE correction parameter and $\mathbf{d}_{i j}(\mathbf{R})$ is the first-order nonadiabatic coupling (NAC) vector.

Previous work demonstrated that replacement of the effective potential term in Eq. (1),

$$
V_{e f f}=\sum_{i}^{F}\left(\frac{1}{2} x_{i}^{2}+\frac{1}{2} p_{i}^{2}-\gamma\right) E_{i}(\mathbf{R})
$$

by a symmetrized one 
$V_{e f f}=\frac{1}{F} \sum_{i}^{F} E_{i}(\mathbf{R})+\frac{1}{F} \sum_{i j}^{F} \frac{1}{2}\left(n_{i}-n_{j}\right)\left(E_{i}(\mathbf{R})-E_{j}(\mathbf{R})\right)$

in the propagation generally gives better dynamics results, ${ }^{82}$ where

$$
n_{i}=\frac{1}{2} x_{i}^{2}+\frac{1}{2} p_{i}^{2}-\gamma
$$

is the action variable for the $i$-th electronic state. Therefore, our current on-the-fly implementation used the following equation of motions (EOMs)

$$
\begin{aligned}
& \frac{d x_{i}}{d t}=p_{i} \frac{1}{F} \sum_{j}^{F}\left(E_{i}(\mathbf{R})-E_{j}(\mathbf{R})\right)+\sum_{j} x_{j} \mathbf{d}_{j i}(\mathbf{R}) \cdot \frac{\mathbf{P}_{\mathrm{kin}}}{\mathbf{M}} \\
& \frac{d p_{i}}{d t}=-x_{i} \frac{1}{F} \sum_{j}^{F}\left(E_{i}(\mathbf{R})-E_{j}(\mathbf{R})\right)+\sum_{j} p_{j} \mathbf{d}_{j i}(\mathbf{R}) \cdot \frac{\mathbf{P}_{\mathrm{kin}}}{\mathbf{M}} \\
& \frac{d \mathbf{R}}{d t}=\frac{\mathbf{P}_{\mathrm{kin}}}{\mathbf{M}} \\
& \frac{d \mathbf{P}_{\mathrm{kin}}}{d t}=-\frac{\partial V_{\mathrm{eff}}}{\partial \mathbf{R}}-\frac{1}{2} \sum_{i j}\left(x_{i} x_{j}+p_{i} p_{j}\right)\left(E_{j}(\mathbf{R})-E_{i}(\mathbf{R})\right) \mathbf{d}_{i j}(\mathbf{R})
\end{aligned}
$$

\subsection{Symmetrical triangle window and initial sampling}

In the SQC/MM dynamics, the "window function" was both used for the initial sampling of the

electronic DoFs and final assignment for the quantum states. The triangle window function ${ }^{91}$ for multistate used in this work is defined as below

$$
W_{i}\left(\mathbf{n}=n_{1}, \ldots n_{i}, \ldots n_{f}\right)=w_{1}\left(n_{i}\right) \cdot \prod_{i \neq j}^{\mathrm{F}} w_{0}\left(n_{i}, n_{j}\right)
$$

with

$$
w_{1}\left(n_{i}\right)=\left\{\begin{array}{cc}
\left(2-\gamma-n_{i}\right)^{2-\mathrm{F}} & \text { for } 1-\gamma<n_{i}<2-\gamma \\
0 & \text { otherwise }
\end{array}\right.
$$

and

$$
w_{0}\left(n_{i}, n_{j}\right)=\left\{\begin{array}{cc}
1 & \text { for } n_{j}<2-2 \gamma-n_{i} \\
0 & \text { otherwise }
\end{array}\right.
$$


The system is regarded as staying at the $i$-th electronic state if $W_{i}=1$ at any moment along the trajectory.

The action-angle sampling method was used to generate the initial $x_{i}^{0}$ and $p_{i}^{0}$ for the $i$-th electronic state, which is

$x_{i}^{0}=\sqrt{2\left(n_{i}^{0}+\gamma\right)} \cos \theta$

$p_{i}^{0}=\sqrt{2\left(n_{i}^{0}+\gamma\right)} \sin \theta$

where $n_{i}^{0}$ is sampled according to the triangle function and $\theta \in[-\pi, \pi]$. The final assignment is performed by binning the action variables into the corresponding windows. In the $\gamma$-fixed approach, this $\gamma$ value is determined by the triangle window function. ${ }^{91}$

\subsection{Trajectory-adjusted electronic ZPE}

Recently, Cotton and Miller proposed a ZPE adjustment protocol, ${ }^{92}$ which modifies the ZPE correction parameter $\gamma$ for each trajectory. After the initial sampling according to the triangle window, the coordinate and momentum of the electronic DoF are $x_{i}^{0}$ and $p_{i}^{0}$, respectively. This in fact defines the ZPE for each trajectory, namely

$\gamma_{i}=\frac{1}{2}\left(x_{i}^{0}\right)^{2}+\frac{1}{2}\left(p_{i}^{0}\right)^{2}-\delta_{i j}$

$j$ is the initial adiabatic electronic state used in the sampling process. This indicates that the corresponding ZPE correction parameter $\gamma_{i}$ of each trajectory is different. Under this trajectoryadjusted $\gamma_{i}$, the action of electronic DoFs becomes 
$n_{i}=\frac{1}{2} x_{i}^{2}+\frac{1}{2} p_{i}^{2}-\gamma_{i}$

during the trajectory propagation. At the same time, the expression of the MM Hamiltonian in the adiabatic representation should also take the trajectory-adjusted ZPE into account, namely

$H=\frac{1}{2 \mathbf{M}} \mathbf{P}_{\text {kin }}^{2}+\sum_{i}^{F}\left(\frac{1}{2} x_{i}^{2}+\frac{1}{2} p_{i}^{2}-\gamma_{i}\right) E_{i}(\mathbf{R})$

In the trajectory propagation, the time-dependent action of the electronic DoFs is calculated according to Eq. (12). Next, the final assignment of the quantum state is performed according to Eq. (7-9) in which the $\gamma$ value is replaced by the trajectory-adjusted $\gamma_{i}$ of each trajectory. More discussions on the technical details of this trajectory-adjusted electronic ZPE were clearly given in previous reference. ${ }^{92}$

\subsection{Computational details}

Both $\mathrm{CH}_{2} \mathrm{NH}_{2}{ }^{+}$and azomethane (Scheme 1) were often taken as typical examples to test the direct nonadiabatic dynamics approaches. ${ }^{30,106,108-121}$ Therefore, we also examined their excited state dynamics by using the on-the-fly SQC/MM dynamics. In addition, the FSSH dynamics were also performed.
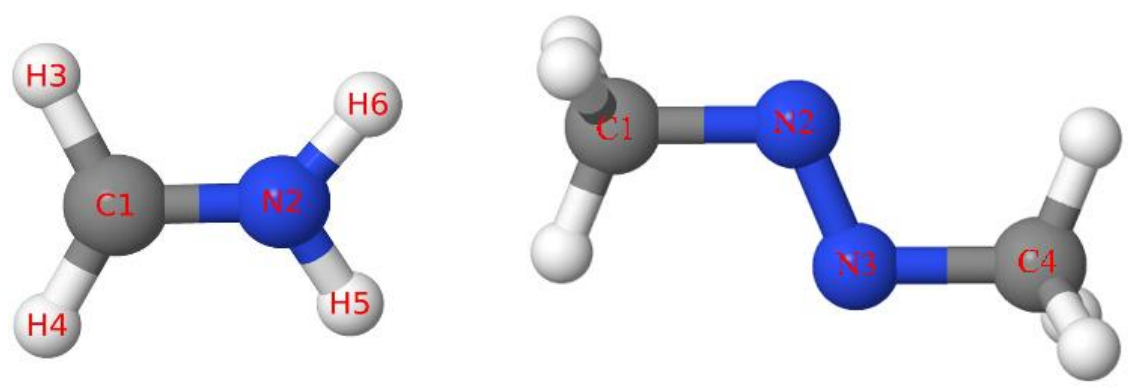

Scheme 1. Chemical structure of $\mathrm{CH}_{2} \mathrm{NH}_{2}{ }^{+}$(left) and azomethane (right) with atom labels. 
The ground state $\left(\mathrm{S}_{0}\right)$ minima were optimized at DFT/B3LYP/6-31G* level using the Gaussian 16 package. $^{122}$ The initial sampling of the nuclear coordinates and momenta was performed by the Wigner sampling of the lowest vibrational level on the electronic ground state. Here the anharmonic effect is neglected for simplicity, and the current Wigner sampling is a practical and standard approach in the on-the-fly nonadiabatic dynamics simulation. To directly compare the dynamics results based on different approaches, the same initial conditions of nuclear coordinates and momenta were used for FSSH, $\gamma$-adjusted and $\gamma$-fixed SQC/MM dynamics.

For $\mathrm{CH}_{2} \mathrm{NH}_{2}{ }^{+}$, we considered the dynamics starting from both the first $\left(\mathrm{S}_{1}\right)$ and second $\left(\mathrm{S}_{2}\right)$ excited state individually. While for azomethane, we explored the dynamics starting from $\mathrm{S}_{1}$.

In the SQC/MM dynamics, the initial sampling and final assignment of the quantum states for electronic DoFs were performed using the symmetrical triangle window tricks. In the SQC/MM dynamics, both $\gamma$-adjusted and $\gamma$-fixed approaches were implemented.

In the FSSH dynamics, the trajectories were first directly put in the adiabatic electronic state where dynamics started. The hop probabilities were calculated according to Tully's Fewest switch algorithm. ${ }^{18}$ We employed the decoherence correction proposed by Granucci et al. and set the parameter $\alpha=0.1 .^{29}$

In the on-the-fly simulation, the electronic structure calculations are performed in each nuclear time step. The electronic motion is generally faster than the nuclear motion. If we use the same time step to integrate the electronic and nuclear motions, the computational cost will become 
extremely high. To reduce it, a standard implementation of on-the-fly TSH dynamics often takes different time steps to propagate the nuclear and electronic motions. For each time step of nuclear motion, a much smaller time step is employed to propagate the electronic motion, and the effective Hamiltonian at each electronic time step is built by the linear interpolation. This approach was clearly discussed in previous works. ${ }^{108,111,123}$ Thus, in the SQC/MM and FSSH dynamics, after several test calculations [see supporting information (SI)], the nuclear equations of motion were integrated with time steps, 0.2 and 0.5 fs, respectively. For each step of nuclear motion, 100 steps of electronic motion were performed. The final results were obtained by averaging over 200 trajectories for all dynamics. The convergence tests with respect to the number of trajectories, nuclear and electronic time steps are given in Figure S1, S2 and S3 (SI), respectively.

The values of electronic energies, first-order derivative NACs and gradients can be obtained from the on-the-fly quantum chemistry calculation. In this work, the complete active space selfconsistent field (CASSCF) method ${ }^{124-125}$ with $6-31 G^{*}$ basis set was used in the electronic structure calculation process. For $\mathrm{CH}_{2} \mathrm{NH}_{2}{ }^{+}$, three state average with active space of six electrons in orbitals [SA-3-CAS(6,4)] was used in the CASSCF calculation. For azomethane, to directly compare with previous AIMS dynamics, ${ }^{120}$ the same state average and active space [SA-2-CAS $\left.(6,4)\right]$ consistent with that in the previous work ${ }^{120}$ were used in the SQC/MM and FSSH dynamics.

All the dynamics calculations were performed with a development version of the JADE package. ${ }^{106-107}$ The CASSCF calculations were performed with the MOLPRO software. ${ }^{126}$ 


\section{Results and Discussion}

\section{1. $\mathrm{CH}_{2} \mathrm{NH}_{2}+$}

\subsubsection{Nonadiabatic dynamics from $S_{2}$}

For $\mathrm{CH}_{2} \mathrm{NH}_{2}{ }^{+}$, all selected dynamics approaches basically give consistent results on the timedependent electronic populations no matter whether the dynamics starts from $S_{2}$ or $S_{1}$, see Figure 1(a) and (b). Here we take $\gamma$-adjusted SQC/MM dynamics as an example. When the dynamics starts from $S_{2}$, the system switches from $S_{2}$ to $S_{1}$ very rapidly (<10 fs), as shown in Figure 1(a). At $10 \mathrm{fs}$, the population in $S_{0}$ starts to increase. The lifetimes of $S_{2}$ and $S_{1}$ are approximately 9 fs and $30 \mathrm{fs}$, respectively, consistent with many previous theoretical works., 2,30,106,108-112,115,118-119
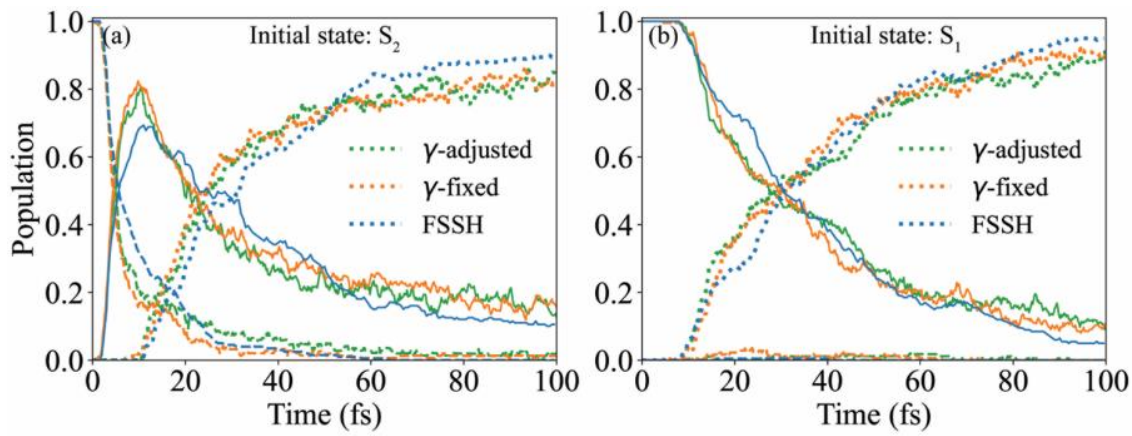

Figure 1. The time-dependent electronic populations of $S_{0}$ (dotted line), $S_{1}$ (solid line) and $S_{2}$ (dashed line) of dynamics staring from (a) $\mathrm{S}_{2}$ and (b) $\mathrm{S}_{1}$ for $\mathrm{CH}_{2} \mathrm{NH}_{2}{ }^{+}$molecule. 200 trajectories were used for all dynamics.

It is well known that the $\mathrm{CN}$ stretching motion plays an important role in the nonadiabatic 
decay dynamics of $\mathrm{CH}_{2} \mathrm{NH}_{2}{ }^{+}$, ${ }^{106,108,112,127}$ when the dynamics starts from $\mathrm{S}_{2}$. To properly show the $\mathrm{CN}$ bond stretching motion in the dynamics starting from $\mathrm{S}_{2}$, we adopted the contour presentation in Figure 2. It is obvious that two types of trajectories (labeled as I and II) exist during the dynamical run. The first group (I) of trajectories displays a significant $\mathrm{CN}$ elongation, while for the second group (II) of trajectories, the $\mathrm{CN}$ bond only experiences weak oscillation patterns. Consistent with previous FSSH results, ${ }^{111}$ we also found that the first group (I) of trajectories show the bi-pyramidalization at both $\mathrm{C}$ and $\mathrm{N}$ site in the $\mathrm{CN}$ elongation process (see below discussion), while the second group (II) of trajectories displays highly mixed motions of the bipyramidalization motions at both $\mathrm{C}$ and $\mathrm{N}$ sites and the torsional motion of the $\mathrm{CN}$ bond. 

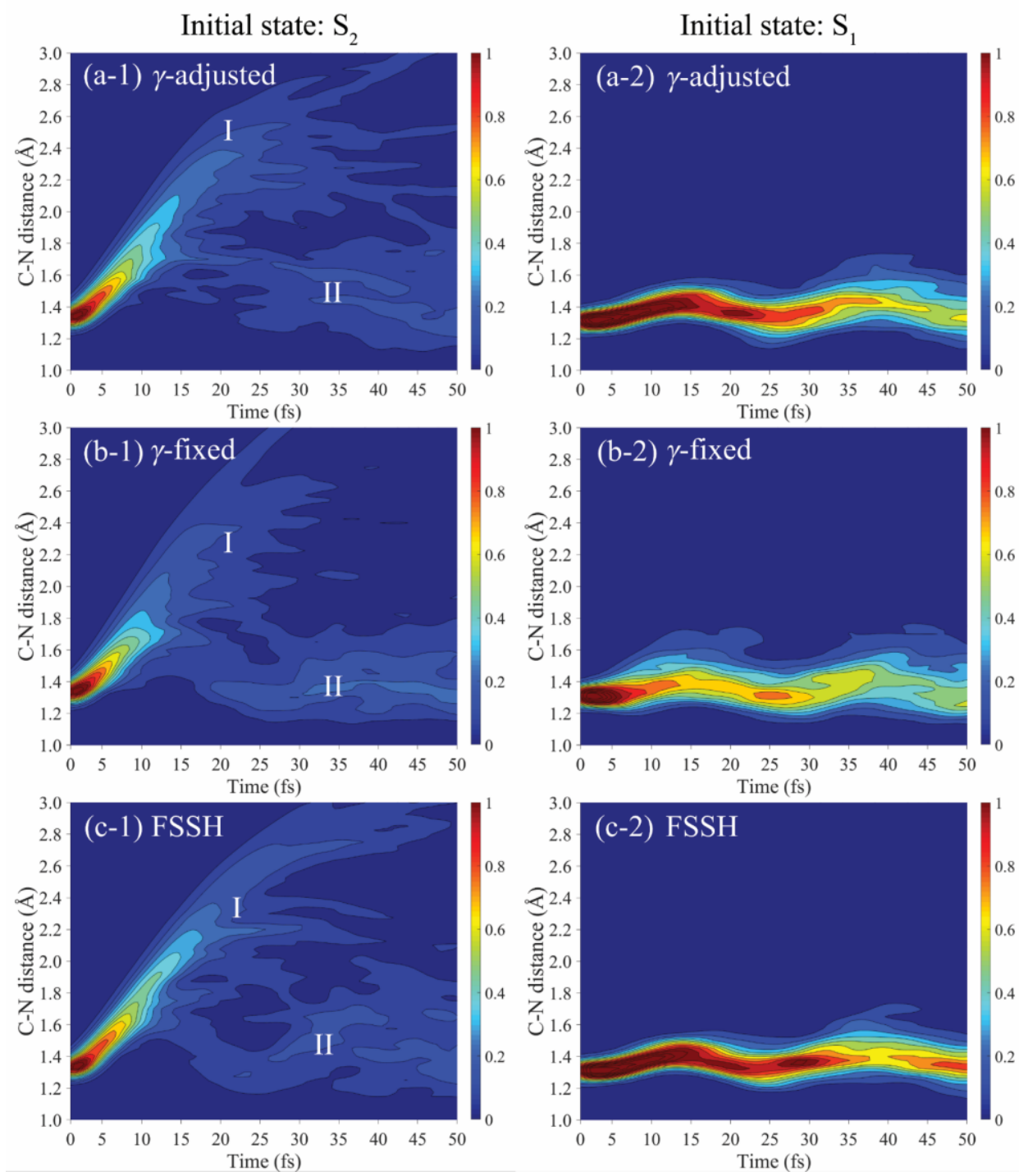

Figure 2. The time-dependent $\mathrm{CN}$ distance distribution of $\mathrm{CH}_{2} \mathrm{NH}_{2}{ }^{+}$in the (a) $\gamma$-adjusted, (b) $\gamma$ fixed SQC/MM and (c) FSSH dynamics. The initial states are (1) $S_{2}$ and (2) $S_{1}$, respectively. 200 trajectories were used for all dynamics. For the dynamics starting from $\mathrm{S}_{2}$, two types of trajectory I and II are given in the subfigures a-1, b-1 and c-1.

It is necessary to know whether the results are different if we only include the data points in 
the windows during the trajectory propagation. We plotted the time-dependent $\mathrm{CN}$ distance distribution under such constraint in Figure S4 (SI), and the results are very similar.

Here we wish to know whether a similar $\mathrm{S}_{0} / \mathrm{S}_{1}$ CI seam is reached or not in the SQC/MM and FSSH dynamics. To perform a more "direct" comparison, we first found the geometry with the minimum $\mathrm{S}_{0} / \mathrm{S}_{1}$ energy gap in the FSSH dynamics, before the trajectory jumped to $\mathrm{S}_{0}$. Next, we tried to extract the geometry with the minimum $\mathrm{S}_{0} / \mathrm{S}_{1}$ energy gap in the SQC/MM dynamics before the trajectory entered the window belong to $\mathrm{S}_{0}$. For all dynamics approaches, we did not consider the trajectory that did not return back to $S_{0}$. The geometrical distributions of these minimumenergy-gap structures were plotted in Figure 3. The key role of the $\mathrm{CN}$ bond stretching motion is confirmed, while the strong bi-pyramidalization motions at both $\mathrm{C}$ and $\mathrm{N}$ sites are also important. For the nonadiabatic dynamics starting from $\mathrm{S}_{2}$, all dynamics approaches show broad distributions of the torsional angles along the $\mathrm{CN}$ motion. However, it is clear that the $\gamma$-adjusted SQC/MM and FSSH dynamics give rather similar descriptions of the $\mathrm{CN}$ stretching motion and the bipyramidalization motion. 

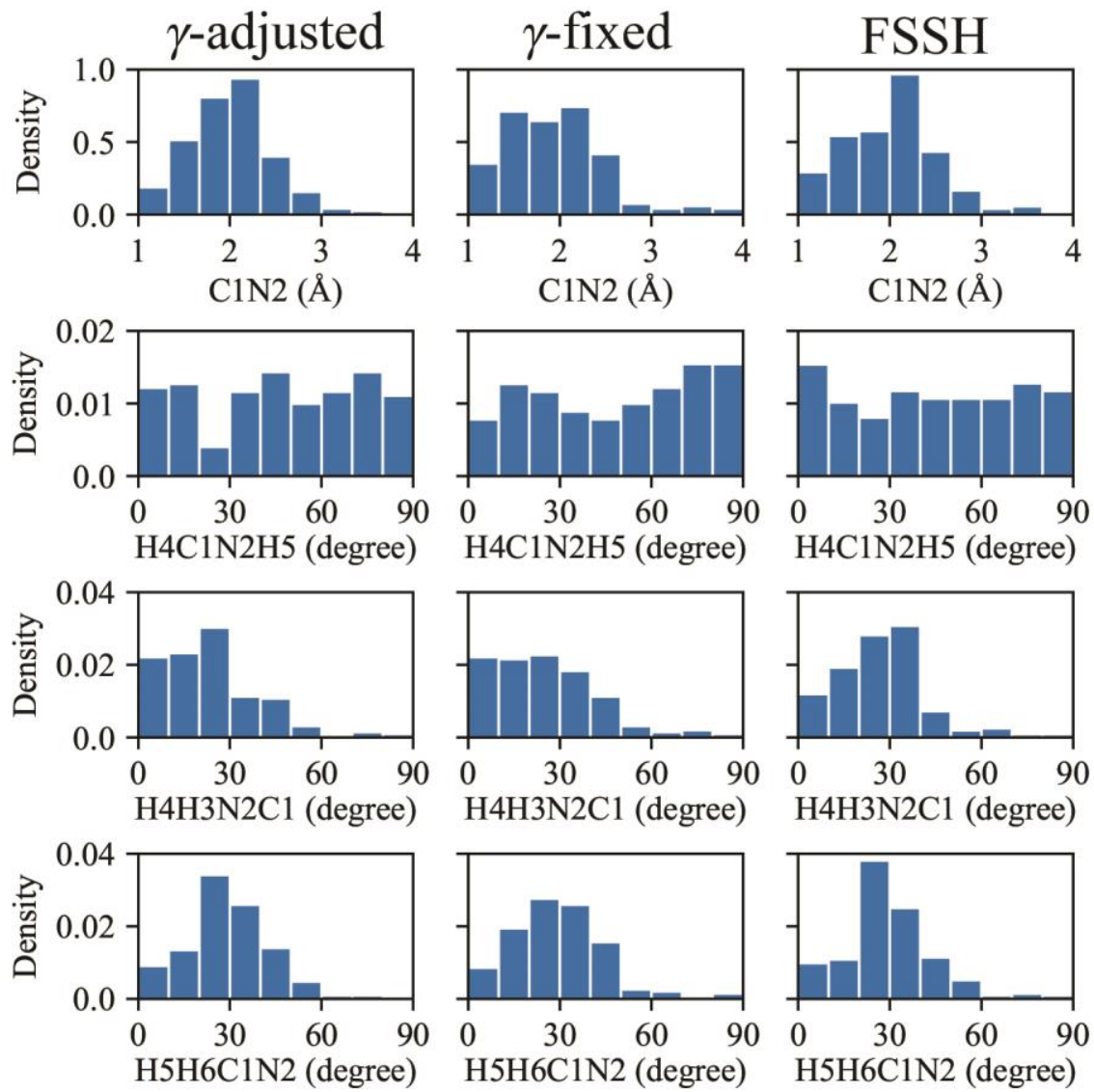

Figure 3. Normalized distribution of key DoFs: bond length C1N2 (CN stretching), dihedral angle $\mathrm{H} 4 \mathrm{C} 1 \mathrm{~N} 2 \mathrm{H} 5$ (CN torsion angle), dihedral angle $\mathrm{H} 4 \mathrm{H} 3 \mathrm{~N} 2 \mathrm{C} 1$ (pyramidalization at $\mathrm{C}$ atom) and dihedral angle $\mathrm{H} 5 \mathrm{H} 6 \mathrm{C} 1 \mathrm{~N} 2$ (pyramidalization at $\mathrm{N}$ atom) of the $\mathrm{CH}_{2} \mathrm{NH}_{2}{ }^{+}$molecule at $\mathrm{S}_{1} / \mathrm{S}_{0}$ minimum-energy-gap structures in $\gamma$-adjusted SQC/MM, $\gamma$-fixed SQC/MM and FSSH dynamics starting from $S_{2}$.

To check whether the single minimum-energy-gap structure in each trajectory can represent the key geometries that play important roles in the nonadiabatic decay process of $\mathrm{CH}_{2} \mathrm{NH}_{2}{ }^{+}$, we also extracted the five geometries with the smallest energy gaps for each trajectory before it 
reached $\mathrm{S}_{0}$ and plotted the distributions of key DoFs in Figure S5 (SI), respectively. The results remain similar.

For the $\mathrm{CH}_{2} \mathrm{NH}_{2}{ }^{+}$nonadiabatic dynamics starting from $\mathrm{S}_{2}$, the nonadiabatic evolutions given by the three dynamics approaches are not far from each other, while some minor differences exist. For instance, the time-dependent populations predicted by the two SQC/MM dynamics are more similar, while slightly different results are obtained in the FSSH dynamics. As a contrast, we notice that the distribution of minimum-energy-gap structures from the $\gamma$-adjusted SQC/MM dynamics is more similar to the FSSH dynamics, while different distribution patterns were found in the $\gamma$-fixed SQC/MM dynamics.

\subsubsection{Nonadiabatic dynamics from $S_{1}$}

When the initial state is $S_{1}$, the exponential decay of $S_{1}$ is observed with a lifetime of $\sim 27 \mathrm{fs}$, see Figure 1(b). During the whole decay dynamics, $S_{2}$ is only weakly populated. Whether to include $S_{2}$ in the dynamics calculations or not does not change the population dynamics significantly, see Figure S6 in SI. It is important to notice that the geometrical evolution in this case is quite different with respect to the dynamics starting from $S_{2}$. For most trajectories, the $\mathrm{CN}$ distance remains relatively short (about 1.2 1.6 ̊) during the evolution, see Figure 2. All dynamics approaches predicted that the bi-pyramidalization at both $\mathrm{C}$ and $\mathrm{N}$ sites, and torsional motion of the $\mathrm{CN}$ bond, are responsible for the $\mathrm{S}_{1} \rightarrow \mathrm{S}_{0}$ decay, as shown in Figure 4. We also noticed that a similar distribution pattern of the minimum-energy gap structures in the trajectory 
propagation was predicted by all dynamics methods.

The same conclusion is drawn when five minimum-energy-gap structures are considered for each trajectory, see Figure S7 in SI.
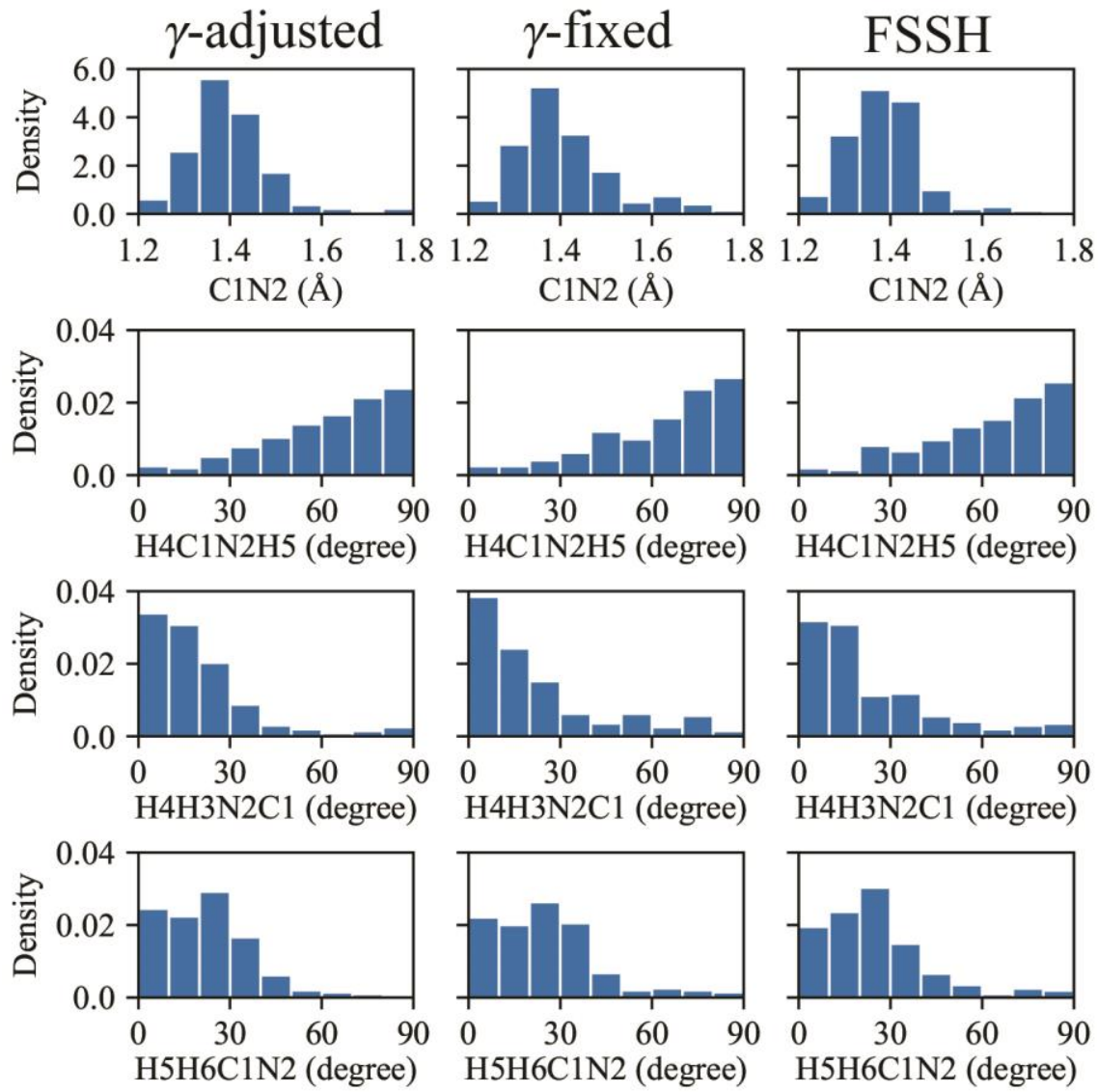

Figure 4. Normalized distributions of key DoFs: bond length C1N2 (CN stretching), dihedral angle $\mathrm{H} 4 \mathrm{C} 1 \mathrm{~N} 2 \mathrm{H} 5$ (CN torsion angle), dihedral angle $\mathrm{H} 4 \mathrm{H} 3 \mathrm{~N} 2 \mathrm{C} 1$ (pyramidalization at $\mathrm{C}$ atom) and dihedral angle $\mathrm{H} 5 \mathrm{H} 6 \mathrm{C} 1 \mathrm{~N} 2$ (pyramidalization at $\mathrm{N}$ atom) of the $\mathrm{CH}_{2} \mathrm{NH}_{2}{ }^{+}$molecule at $\mathrm{S}_{1} / \mathrm{S}_{0}$ minimum-energy-gap structures in $\gamma$-adjusted SQC/MM, $\gamma$-fixed SQC/MM and FSSH dynamics starting from $S_{1}$. 
No matter whether the dynamics starts from $S_{1}$ or $S_{2}$, the dynamics features, including excited-state lifetimes, the major reactive molecular motion in the excited-state nonadiabatic dynamics, and the geometrical feature in the relevant CI seam are similar in the description by different dynamics methods used in this work, the $\gamma$-adjusted and $\gamma$-fixed SQC/MM and FSSH, see Figure 1-4. In addition, the dynamics results are highly consistent with previous on-the-fly studies with various methods, such as the TSH dynamics, ${ }^{106,108-112,118,128}$ and the more rigorous TSH approach from the exact factorization of electronic-nuclear wavefunction. ${ }^{30}$

Both SQC/MM and FSSH approaches should be rather important in the on-the-fly dynamics community. In the current work, the dynamics results from SQC/MM and FSSH are very similar for $\mathrm{CH}_{2} \mathrm{NH}_{2}{ }^{+}$, while these results are not trivial and contain important meanings. For instance, this work confirms that the on-the-fly SQC/MM dynamics can be used to describe the nonadiabatic dynamics of $\mathrm{CH}_{2} \mathrm{NH}_{2}{ }^{+}$, a prototype system to test the on-the-fly nonadiabatic dynamics methods. This gives us the confidence to use this approach in the further study of realistic systems. At the same time, the current work also gives additional evidence to support the previous understanding of the excited-state process of $\mathrm{CH}_{2} \mathrm{NH}_{2}{ }^{+}$by the TSH method, ${ }^{106,108-112,118,128}$ making sure that the proper description of the nonadiabatic dynamics of $\mathrm{CH}_{2} \mathrm{NH}_{2}{ }^{+}$is achieved. In fact, this type of comparison is very important for the general on-the-fly simulation. Only when different dynamics simulations give consistent results may we get the confidence that the nonadiabatic dynamics is properly described theoretically. 


\subsection{Azomethane}

Azomethane is often used as a chemical source for radical generation since it can decompose into alkyl radicals and nitrogen after absorption of ultraviolet light in the gas phase. ${ }^{129}$ Previous theoretical studies with the FSSH and AIMS methods showed that azomethane would undergo an ultrafast nonadiabatic decay process after excitation. ${ }^{120-121}$ As a typical system for benchmark, we chose azomethane to examine the SQC/MM dynamics. Here we used the CASSCF level with SA2-CAS $(6,4)$ in the SQC/MM and FSSH dynamics, and the same electronic structure level was employed in previous AIMS work. ${ }^{120}$

For azomethane, only two states, $\mathrm{S}_{1}$ and $\mathrm{S}_{0}$, are involved in the nonadiabatic dynamics process in this work. When the systems are put in $\mathrm{S}_{1}$, most of the trajectories decay to $\mathrm{S}_{0}$ within $350 \mathrm{fs}$ for $\gamma$-adjusted SQC/MM dynamics, consistent with our own FSSH dynamics and previous AIMS dynamics. ${ }^{120-121}$ While for the $\gamma$-fixed SQC/MM dynamics, about half of the trajectories still stay at $\mathrm{S}_{1}$ at $350 \mathrm{fs}$, see Figure 5(a). This phenomenon is very unlike that of $\mathrm{CH}_{2} \mathrm{NH}_{2}{ }^{+}$, where the timedependent electronic populations of $\mathrm{S}_{1}$ of SQC/MM dynamics with both $\gamma$-adjusted and $\gamma$-fixed versions are very close (Figure 1). 

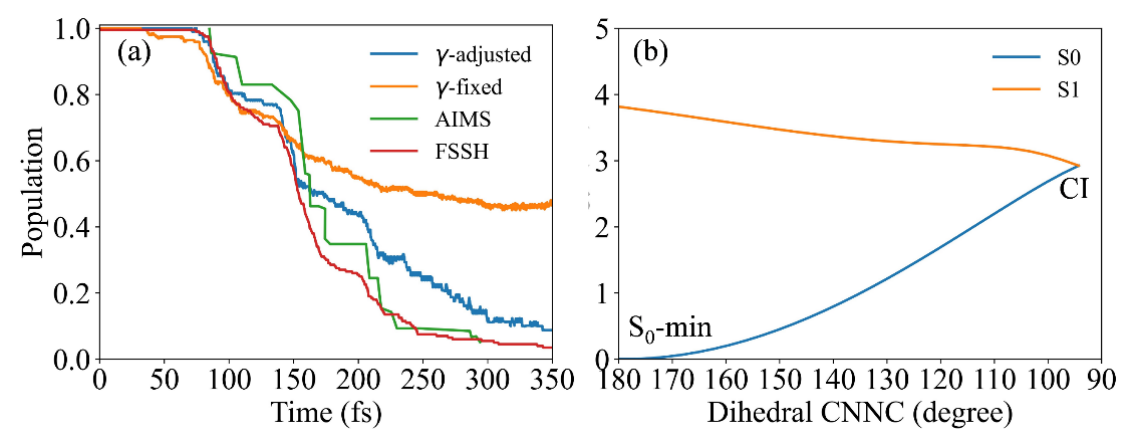

Figure 5. (a): Time-dependent electronic populations of $S_{1}$ with different dynamics methods. 200 trajectories are used in the SQC/MM and FSSH dynamics. The results of the AIMS dynamics are taken from previous work. ${ }^{120}$ (b) Linear interpolated potential energy curve from $\mathrm{S}_{0}$ minimum to CI.

To understand the general feature of nonadiabatic dynamics of azomethane, we optimized the $\mathrm{S}_{1} / \mathrm{S}_{0} \mathrm{CI}$, and plotted the linear interpolated potential energy curve from $\mathrm{S}_{0}$ minimum to $\mathrm{CI}$, see Figure 5(b). Consistent with previous studies, ${ }^{120}$ there is no barrier along the photoisomerization pathway.

Since the rotation of the central N-N double bond, characterized by the dihedral angle of CNNC, plays an important role in the nonadiabatic decay of azomethane, the time-dependent evolution of dihedral angle of CNNC for all trajectories of SQC/MM dynamics with $\gamma$-adjusted and $\gamma$-fixed is plotted, see Figure 6. The dihedral angles after the minimum-energy-gap structures are not plotted for a better view of the trajectory propagation on the excited state from the FranckCondon (FC) region to the $\mathrm{S}_{1} / \mathrm{S}_{0} \mathrm{CI}$. 

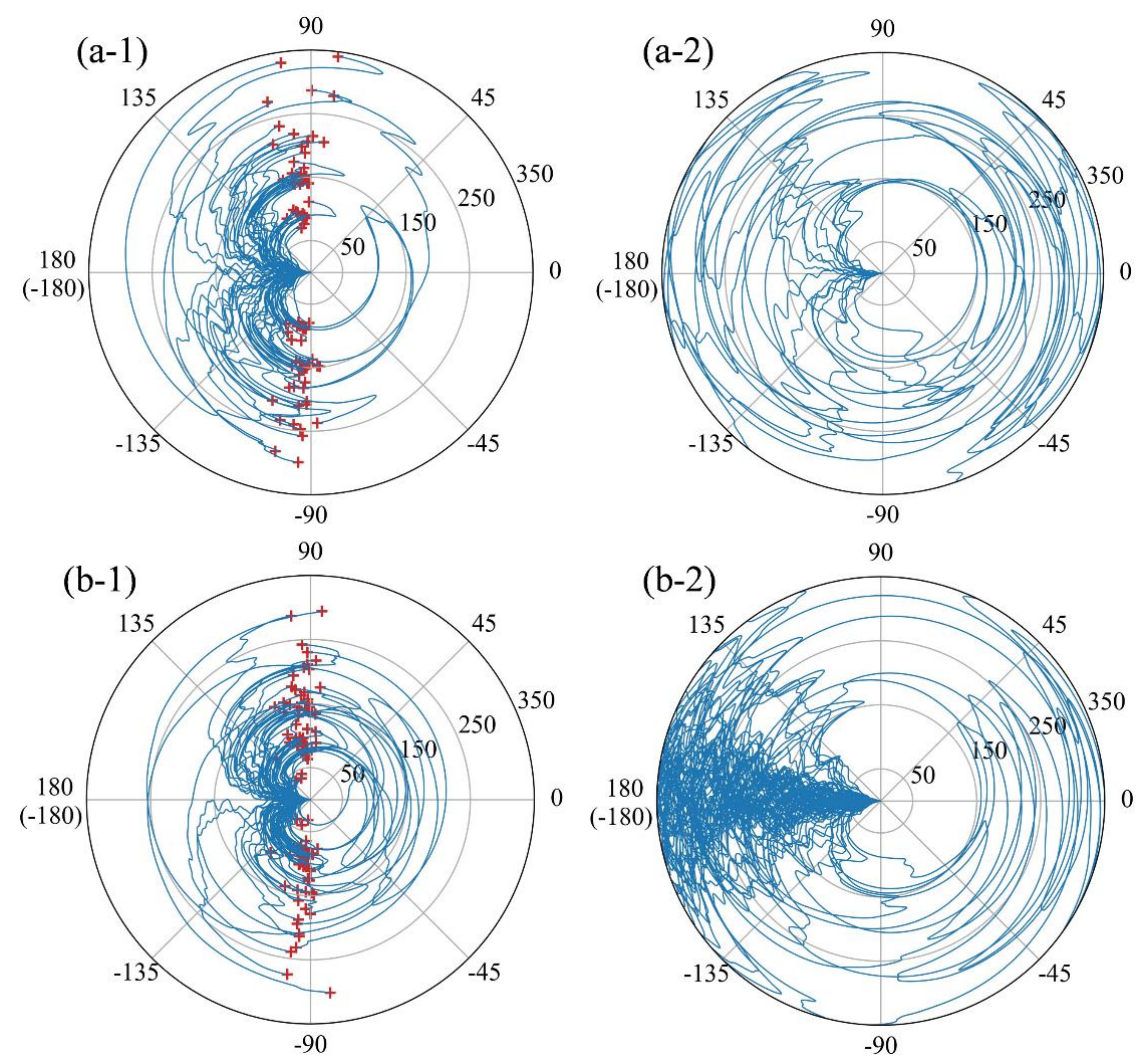

Figure 6. Time-dependent evolutions of dihedral angle of CNNC for all trajectories before decaying to $\mathrm{S}_{0}$ of (a) $\gamma$-adjusted and (b) $\gamma$-fixed SQC/MM dynamics. The trajectories that decay and do not decay to the $S_{0}$ within 350 fs are shown in (1) and (2) separately. The red points indicate the $\mathrm{S}_{1} / \mathrm{S}_{0}$ minimum-energy-gap structures. The radial and angular in the polar coordinates represent the time (fs) and dihedral angle (degree), respectively.

Clearly, the trajectory must experience a strong twisting motion of the CNNC dihedral angles if it goes to $S_{0}$ via the $S_{0} / S_{1} C I$. Otherwise, the trajectory remains on the excited state, and no $S_{1}$ population decays observed. For $\gamma$-adjusted dynamics, most trajectories show the strong rotation of the NN double bond, and the trajectory can access to these geometries with a very small energy 
gap. This means that the majority of the trajectories follow the isomerization channels and decay to $\mathrm{S}_{0}$ via the relevant CIs. While for $\gamma$-fixed dynamics, a large number of trajectories do not show the significant CNNC twisting motion, but display an oscillation of the CNNC angle around 180 (-180) degrees. These trajectories simply stay on $S_{1}$ and do not decay to $S_{0}$ at all.

Figure $5(b)$ shows that the energy of the $S_{0} / S_{1} C I$ is lower than that of $S_{1}$ at the $S_{0}$-min geometry. No energy barrier exists along the isomerization reaction pathway. Thus, the trajectory should naturally follow the barrierless pathway and access the $\mathrm{S}_{0} / \mathrm{S}_{1} \mathrm{CI}$ without any doubt. However, for $\gamma$-fixed dynamics, a large number of trajectories seem to just stay in $\mathrm{S}_{1}$. The problematic feature can be explained by the fact that the nuclear motion is propagated by a kind of mean potential energy surface (PES), instead of $\mathrm{S}_{1}$ PES even before the trajectory reaches the $\mathrm{S}_{0} / \mathrm{S}_{1} \mathrm{CI}$. In $\gamma$-fixed dynamics, both $\mathrm{S}_{1}$ and $\mathrm{S}_{0}$ were involved in the nonadiabatic dynamics of azomethane, and the nuclear motion is governed by the effective potential

$V_{e f f}=n_{0} E_{0}(\mathbf{R})+n_{1} E_{1}(\mathbf{R})$

where

$$
\begin{aligned}
& n_{0}=\frac{1}{2}\left(\left(x_{0}\right)^{2}+\left(p_{0}\right)^{2}\right)-\gamma \\
& n_{1}=\frac{1}{2}\left(\left(x_{1}\right)^{2}+\left(p_{1}\right)^{2}\right)-\gamma
\end{aligned}
$$

are time-dependent action variables of electronic states for each trajectory. Please notice that the effective potential on the nuclear part given in Eq. (14) is rather approximated when the symmetrized form of the effective potential [Eq. (4)] is employed. For each single trajectory, only when $n_{0}+n_{1}=1$, Eq. (4) becomes completely equivalent to Eq. (14). However, this condition is not 
always true for each single trajectory since $n_{0}$ and $n_{1}$ are sampled independently in standard $\gamma$ fixed SQC dynamics. This feature was carefully discussed in detail by several previous works. ${ }^{77-}$ ${ }^{78,92}$ However, the essential ideas behind them should be rather similar and thus we still can use Eq. (14-15) for the below discussions.

Certainly, the effective potential given in Eq. (14) defines the nuclear motion at the very beginning of the dynamics. Because both two action variables were obtained stochastically by sampling procedure, the effective potential is the linear combination of the $S_{0}$ and $S_{1}$ potential. As a consequence, the $\mathrm{BO}$ dynamics is not recovered for the single trajectory, while in principle the early-stage dynamics should follow the $S_{1}$ surface due to the negligible $S_{0} / S_{1} N A C$ in the FC region. In the azomethane, $S_{0}$ is a strong bounded state near the $S_{0}$ minimum geometry. If the contribution of the $\mathrm{n}_{0}$ is large enough, the effective potential may become a bounded potential that prevents the trajectory escaping from the FC region. To clarify this idea, we plotted the initial sampled action variables and labeled their final assignment in Figure 7. 


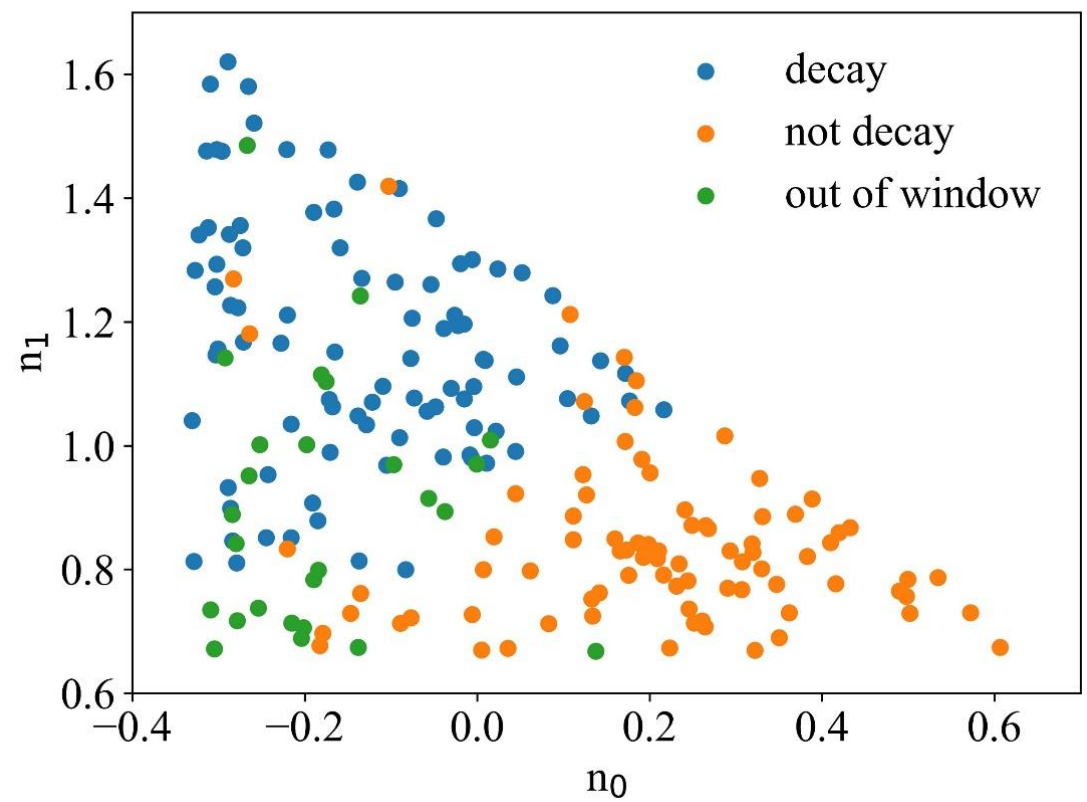

Figure 7. Distribution of the initial sample $n_{0}$ and $n_{1}$ of all trajectories for $\gamma$-fixed dynamics. The labels "decay" and "not decay" mean the trajectory decays and does not decay to $\mathrm{S}_{0}$ within 350 fs in the $\gamma$-fixed SQC/MM dynamics, respectively. The label "out of window" means the action values of the trajectory are not in any triangle windows at the end of dynamics.

It is clear that the trajectories are more likely to stay in the excited state when $n_{0}$ is large. In this condition, the effective PES might become bounded, and the trajectory cannot arrive at the CI region. This explains why many trajectories do not decay in Figure 5(a).

As a contrast, in the $\gamma$-adjusted situation, the ZPE correction value of each trajectory is determined by its own initial condition. In such a case, the early-time dynamics almost becomes the pure BO dynamics when the NAC is small enough under the large $\mathrm{S}_{0} / \mathrm{S}_{1}$ energy gap. In this way, most trajectories may follow the correct potential and access the CI. This explains the fast 
and completed population decay of the $\gamma$-adjusted SQC/MM dynamics in Figure 5(a).

Clearly, the $\gamma$-adjusted SQC/MM dynamics result is much closer to that of the AIMS dynamics in Figure 5(a), ${ }^{120}$ we suggest that this $\gamma$-adjusted approach should always be used. In addition, this choice is consistent with physical insight. When the ground state and excited states are well separated in the energy domain in the FC region, the early-time excited-state dynamics should essentially the BO dynamics due to the extremely weak NAC. Thus, the employment of the $\gamma$-adjusted approach allows the trajectory propagation to recover this limit. This avoids the possible deficiency of the effective potential acting on the nuclear propagation in the $\gamma$-fixed approach. This observation is consistent with the ideas by Cotton and Miller, ${ }^{92}$ who strongly suggested using the $\gamma$-adjusted approach in the study of the gas-phase photochemistry of the molecular systems without too many DoFs.

\subsection{Discussions}

Here we wish to give a few of additional comments and discussions on the current works.

The understanding of the excited-state nonadiabatic dynamic is rather challenging for the realistic molecules. Many ultrafast spectroscopic experiments give time-resolved spectra. In order to understand the nonadiabatic dynamics and analyze such spectra, theoretical simulations are necessary. To describe the realistic systems with many DoFs and arbitrary PESs, the on-the-fly nonadiabatic dynamics simulation becomes irreplaceable. Different electronic methods have different advantages and shortcomings, and previous works showed that the on-the-fly dynamics 
with different electronic structure methods may give different results. ${ }^{107,130}$ In principle, the same situations exist for the nonadiabatic dynamics methods.

For surface hopping dynamics, the over-decoherence problem is well known. ${ }^{19-20,29}$ Although many theoretical approaches were proposed to add the decoherence corrections and many on-thefly implementation works used them for practical reasons, ${ }^{19-21,29,106,127,131}$ many corrections are rather phenomenological and the empirical parameters are often added. The underlying reason is that researchers have still not found a formal way to derive the surface hopping dynamics from rigorous semiclassical approximations. Thus, the improvement of the performance of surface hopping dynamics is rather challenging. As a contrast, the SQC/MM dynamics can more-or-less capture the electronic coherence without the inclusion of empirical corrections. ${ }^{81,86}$ More importantly, the SQC/MM approach can be improved systematically by adding more quantum corrections based on more rigorous semiclassical dynamics. In the SQC/MM dynamics, the trajectories are independently propagated, and thus the computational cost is in principle smaller than that of the wavepacket-based methods, as like AIMS and vMCG.

Only when we combine various dynamics approaches and different electronic structure methods in the on-the-fly simulation, it is possible to construct the correct and comprehensive understandings of the nonadiabatic procedures of polyatomic systems. For example, when the onthe-fly simulations with different dynamics and electronic structure methods give consistent results, we more or less believe that the proper description of the nonadiabatic dynamics is achieved. This situation is observed in our simulation of $\mathrm{CH}_{2} \mathrm{NH}_{2}{ }^{+}$. Certainly, when the results are strongly 
dependent on the selected dynamics methods, more additional theoretical and experimental studies should be performed. In addition, when different problems were concerned, it may be suitable to choose the proper methods. Thus, it is always worthwhile to implement different dynamics approaches in the on-the-fly simulation. Previous works already demonstrated that the SQC/MM dynamics is a rather promising approach, ${ }^{38,73-90}$ so it is highly preferable to introduce it in the onthe-fly simulation.

In the $\gamma$-fixed SQC/MM dynamics, we employed the triangle window proposed by Miller and Cotton. ${ }^{80,91}$ They recommended the triangle window, because it gives a similar description for normal nonadiabatic dynamics compared with the SQC/MM dynamics with the rectangle window, while it gives a better description in the weak diabatic coupling situation. In such work, they also strongly suggest using $\gamma=1 / 3$ in the application of the triangle window due to the geometrical reason. ${ }^{80,91}$ Here we preferred to use this ZPE correction instead of modifying its value due to the below reasons.

For the $\gamma$-fixed SQC/MM dynamics with the square window, several works clearly demonstrated that the results are strongly dependent on the selection of the $\gamma$ value. ${ }^{77,105}$ In principle, a similar conclusion should be reached in the on-the-fly implementation of this approach. However, in the on-the-fly simulation, it is not possible to choose the $\gamma$ value by running many preliminary calculations because of the huge computational costs and the lack of accurate results. In addition, a too narrow window may give better results for anharmonic systems, ${ }^{77}$ while in this setup, a large number of trajectories go out of windows and it is not easy to converge calculations. 
In this sense, it is more important to select a reasonable $\gamma$ value within a proper window before the treatment of realistic systems. Since the $\gamma$ value can be derived by geometry feature for the triangle window, ${ }^{80,91}$ we think that it is suitable to just take it instead of adjusting its value.

At the same time, we also realize that the $\gamma$-adjusted SQC/MM dynamics always give much better dynamics, particularly for the second test system. This already confirms that we should anyway use the $\gamma$-adjusted SQC/MM dynamics in the future on-the-fly simulation. Thus the examination of the influence of the $\gamma$ value for the $\gamma$-fixed SQC/MM dynamics is not essential in the current work.

\section{Conclusion}

In this work, we tried to implement the on-the-fly SQC/MM dynamics. The SQC/MM dynamics formulism in the adiabatic representation provides a rather clear idea for the direct combination with the electronic structure calculations. We chose $\mathrm{CH}_{2} \mathrm{NH}_{2}{ }^{+}$and azomethane as typical examples to examine the performance of the on-the-fly SQC/MM dynamics. The results show that all dynamical features of the nonadiabatic dynamics of $\mathrm{CH}_{2} \mathrm{NH}_{2}{ }^{+}$are well captured by the on-the-fly SQC/MM dynamics, no matter the $\gamma$-adjusted or $\gamma$-fixed approach is used. While for azomethane, the $\gamma$-adjusted SQC/MM dynamics performs much better than the $\gamma$-fixed SQC/MM dynamics. The reason is that the former one can recover the BO dynamics features at the beginning of the trajectory when the electronic states are far from each other, while the latter one always uses 
a kind of mean PES. As a result, the $\gamma$-adjusted approach is strongly recommended to be used in all on-the-fly SQC/MM nonadiabatic dynamics.

This work demonstrates that the current on-the-fly SQC/MM dynamics provides a very promising approach to simulate the nonadiabatic dynamics of polyatomic molecules. When the MM Hamiltonian is considered, the SQC/MM dynamics is a simple dynamics approach, which serves as a zero-order approximation of more advanced semi-classical dynamics methods with the inclusion of quantum effects. Starting from the SQC/MM dynamics, it is possible to obtain other sophisticated semi-classical methods with different accurate levels by introducing more quantum correction terms. Thus, the current implementation work provides a good starting point to cooperate with more rigorous semi-classical dynamics in the on-the-fly dynamics simulation.

\section{ASSOCIATED CONTENT}

\section{Supporting Information}

This information is available free of charge via the Internet at http://pubs.acs.org

Convergence tests of the number of trajectories, time steps of nuclear and electronic motions, time-dependent $\mathrm{CN}$ distance distribution spanned by trajectories in the window, key DoF distributions of five $\mathrm{S}_{1} / \mathrm{S}_{0}$ minimum-energy-gap structures and time-dependent electronic populations with and without $\mathrm{S}_{2}$ in the dynamics starting from $\mathrm{S}_{1}$. 


\section{Author Information}

\section{Corresponding Author}

E-mail: zhenggang.lan@m.scnu.edu.cn; zhenggang.lan@gmail.com.

\section{Author Contributions}

${ }^{\circ}$ Deping $\mathrm{Hu}$ and $\mathrm{Yu}$ Xie contributed equally to this work.

\section{Notes}

The authors declare no competing financial interest.

\section{Acknowledgments}

This work is supported by NSFC projects (No. 21933011, 21873112 and 21673266). The authors thank the Supercomputing Center, Computer Network Information Center, Chinese Academy of Sciences; National Supercomputing Center in Shenzhen for providing computational resources.

\section{References}

(1) Domcke, W.; Yarkony, D. R.; Köppel, H., Conical intersections: Electronic structure, dynamics and spectroscopy. World Scientic: 2004.

(2) Domcke, W.; Yarkony, D. R.; Köppel, H., Conical intersections: Theory, computation and experiment. World Scientific: 2011.

(3) May, V.; Kühn, O., Charge and energy transfer dynamics in molecular systems. Wiley-VCH Verlag GmbH \& Co.: KGaA,Boschstr. 12, 69469 Weinheim, Germany, 2011.

(4) Schröter, M.; Ivanov, S. D.; Schulze, J.; Polyutov, S. P.; Yan, Y.; Pullerits, T.; Kühn, O. Excitonvibrational coupling in the dynamics and spectroscopy of Frenkel excitons in molecular aggregates. Phys. Rep. 2015, 567, 1-78. 
(5) Curchod, B. F. E.; Martínez, T. J. Ab initio nonadiabatic quantum molecular dynamics. Chem. Rev. 2018, $118,3305-3336$.

(6) Yonehara, T.; Hanasaki, K.; Takatsuka, K. Fundamental approaches to nonadiabaticity: Toward a chemical theory beyond the Born-Oppenheimer paradigm. Chem. Rev. 2012, 112, 499-542.

(7) Akimov, A. V.; Neukirch, A. J.; Prezhdo, O. V. Theoretical insights into photoinduced charge transfer and catalysis at oxide interfaces. Chem. Rev. 2013, 113, 4496-4565.

(8) Stock, G.; Thoss, M. Classical description of nonadiabatic quantum dynamics. Adv. Chem. Phys. 2005, 131, 243-375.

(9) Crespo-Otero, R.; Barbatti, M. Recent advances and perspectives on nonadiabatic mixed quantumclassical dynamics. Chem. Rev. 2018, 118, 7026-7068.

(10) Ben-Nun, M.; Martínez, T. J. Ab initio quantum molecular dynamics. Adv. Chem. Phys. 2002, $121,439-512$.

(11) Beck, M. H.; Jackle, A.; Worth, G. A.; Meyer, H. D. The multiconfiguration time-dependent Hartree (MCTDH) method: A highly efficient algorithm for propagating wavepackets. Phys. Rep. 2000, 324, 1-105.

(12) Wang, H. B.; Thoss, M. Multilayer formulation of the multiconfiguration time-dependent hartree theory. J. Chem. Phys. 2003, 119, 1289-1299.

(13) Yao, Y.; Sun, K.-W.; Luo, Z.; Ma, H. Full quantum dynamics simulation of a realistic molecular system using the adaptive time-dependent density matrix renormalization group method. J. Phys. Chem. Lett. 2018, 9, 413-419.

(14) Greene, S. M.; Batista, V. S. Tensor-train split-operator Fourier transform (TT-SOFT) method: Multidimensional nonadiabatic quantum dynamics. J. Chem. Theory Comput. 2017, 13, 40344042.

(15) Ren, J. J.; Shuai, Z. G.; Chan, G. K. L. Time-dependent density matrix renormalization group algorithms for nearly exact absorption and fluorescence spectra of molecular aggregates at both zero and finite temperature. J. Chem. Theory Comput. 2018, 14, 5027-5039. 
(16) Li, X.; Tully, J. C.; Schlegel, H. B.; Frisch, M. J. Ab initio Ehrenfest dynamics. J. Chem. Phys. 2005, 123, 084106.

(17) Zhu, C. Y.; Jasper, A. W.; Truhlar, D. G. Non-Born-Oppenheimer trajectories with self-consistent decay of mixing. J. Chem. Phys. 2004, 120, 5543-5557.

(18) Tully, J. C. Molecular-dynamics with electronic-transitions. J. Chem. Phys. 1990, 93, 1061-1071.

(19) Subotnik, J. E.; Jain, A.; Landry, B.; Petit, A.; Ouyang, W. J.; Bellonzi, N. Understanding the surface hopping view of electronic transitions and decoherence. Annu. Rev. Phys. Chem. 2016, 67, $387-417$.

(20) Wang, L. J.; Akimov, A.; Prezhdo, O. V. Recent progress in surface hopping: 2011-2015. J. Phys. Chem. Lett. 2016, 7, 2100-2112.

(21) Mai, S.; Marquetand, P.; González, L. Nonadiabatic dynamics: The SHARC approach. Wiley Interdiscip. Rev.: Comput. Mol. Sci. 2018, 8, e1370.

(22) Chen, H. T.; Reichman, D. R. On the accuracy of surface hopping dynamics in condensed phase non-adiabatic problems. J. Chem. Phys. 2016, 144, 094104.

(23) Granucci, G.; Persico, M.; Zoccante, A. Including quantum decoherence in surface hopping. $J$. Chem. Phys. 2010, 133, 134111.

(24) Zhu, C. Y.; Nobusada, K.; Nakamura, H. New implementation of the trajectory surface hopping method with use of the Zhu-Nakamura theory. J. Chem. Phys. 2001, 115, 3031-3044.

(25) Zimmermann, T.; Vaníček, J. Efficient on-the-fly ab initio semiclassical method for computing time-resolved nonadiabatic electronic spectra with surface hopping or Ehrenfest dynamics. J. Chem. Phys. 2014, 141, 134102.

(26) Begušić, T.; Roulet, J.; Vaníček, J. On-the-fly ab initio semiclassical evaluation of time-resolved electronic spectra. J. Chem. Phys. 2018, 149, 244115.

(27) Persico, M.; Granucci, G. An overview of nonadiabatic dynamics simulations methods, with focus on the direct approach versus the fitting of potential energy surfaces. Theor. Chem. Acc. 2014, 133, 1526. 
(28) Belyaev, A. K.; Lasser, C.; Trigila, G. Landau-Zener type surface hopping algorithms. J. Chem. Phys. 2014, 140, 224108.

(29) Granucci, G.; Persico, M. Critical appraisal of the fewest switches algorithm for surface hopping. J. Chem. Phys. 2007, 126, 134114.

(30) Ha, J.-K.; Lee, I. S.; Min, S. K. Surface hopping dynamics beyond nonadiabatic couplings for quantum coherence. J. Phys. Chem. Lett. 2018, 9, 1097-1104.

(31) Abedi, A.; Maitra, N. T.; Gross, E. K. U. Exact factorization of the time-dependent electronnuclear wave function. Phys. Rev. Lett. 2010, 105, 123002.

(32) Richings, G. W.; Polyak, I.; Spinlove, K. E.; Worth, G. A.; Burghardt, I.; Lasorne, B. Quantum dynamics simulations using Gaussian wavepackets: The vMCG method. Int. Rev. Phys. Chem. 2015, 34, 269-308.

(33) Shalashilin, D. V. Quantum mechanics with the basis set guided by Ehrenfest trajectories: Theory and application to spin-boson model. J. Chem. Phys. 2009, 130, 244101.

(34) Makhov, D. V.; Symonds, C.; Fernandez-Alberti, S.; Shalashilin, D. V. Ab initio quantum direct dynamics simulations of ultrafast photochemistry with Multiconfigurational Ehrenfest approach. Chem. Phys. 2017, 493, 200-218.

(35) Symonds, C.; Kattirtzi, J. A.; Shalashilin, D. V. The effect of sampling techniques used in the multiconfigurational Ehrenfest method. J. Chem. Phys. 2018, 148, 184113.

(36) Meyer, H. D.; Miller, W. H. Classical analog for electronic degrees of freedom in non-adiabatic collision processes. J. Chem. Phys. 1979, 70, 3214-3223.

(37) Cotton, S. J.; Miller, W. H. Symmetrical windowing for quantum states in quasi-classical trajectory simulations. J. Phys. Chem. A 2013, 117, 7190-7194.

(38) Miller, W. H.; Cotton, S. J. Classical molecular dynamics simulation of electronically nonadiabatic processes. Faraday Discuss. 2016, 195, 9-30.

(39) Stock, G.; Thoss, M. Semiclassical description of nonadiabatic quantum dynamics. Phys. Rev. Lett. 1997, 78, 578-581. 
(40) Liu, J. A unified theoretical framework for mapping models for the multi-state Hamiltonian. $J$. Chem. Phys. 2016, 145, 204105.

(41) Sun, X.; Miller, W. H. Semiclassical initial value representation for electronically nonadiabatic molecular dynamics. J. Chem. Phys. 1997, 106, 6346-6353.

(42) Saller, M. A. C.; Kelly, A.; Richardson, J. O. On the identity of the identity operator in nonadiabatic linearized semiclassical dynamics. J. Chem. Phys. 2019, 150, 071101.

(43) Saller, M. A. C.; Kelly, A.; Richardson, J. O. Improved population operators for multi-state nonadiabatic dynamics with the mixed quantum-classical mapping approach. Faraday Discuss. 2020, 221, 150-167.

(44) He, X.; Liu, J. A new perspective for nonadiabatic dynamics with phase space mapping models. J. Chem. Phys. 2019, 151, 024105.

(45) Mulvihill, E.; Gao, X.; Liu, Y. D.; Schubert, A.; Dunietz, B. D.; Geva, E. Combining the mapping hamiltonian linearized semiclassical approach with the generalized quantum master equation to simulate electronically nonadiabatic molecular dynamics. J. Chem. Phys. 2019, 151, 074103.

(46) Liu, Y.; Gao, X.; Lai, Y.; Mulvihill, E.; Geva, E. Electronic dynamics through conical intersections via quasiclassical mapping Hamiltonian methods. J. Chem. Theory Comput. 2020, $16,4479-4488$.

(47) Gao, X.; Saller, M. A. C.; Liu, Y.; Kelly, A.; Richardson, J. O.; Geva, E. Benchmarking quasiclassical mapping Hamiltonian methods for simulating electronically nonadiabatic molecular dynamics. J. Chem. Theory Comput. 2020, 16, 2883-2895.

(48) Gao, X.; Lai, Y.; Geva, E. Simulating absorption spectra of multiexcitonic systems via quasiclassical mapping Hamiltonian methods. J. Chem. Theory Comput. 2020, 16, 6465-6480.

(49) Gao, X.; Geva, E. A nonperturbative methodology for simulating multidimensional spectra of multiexcitonic molecular systems via quasiclassical mapping Hamiltonian methods. J. Chem. Theory Comput. 2020, 16, 6491-6502.

(50) Kim, H.; Nassimi, A.; Kapral, R. Quantum-classical Liouville dynamics in the mapping basis. $J$. 
Chem. Phys. 2008, 129, 084102.

(51) Kelly, A.; Rhee, Y. M. Mixed quantum-classical description of excitation energy transfer in a model Fenna-Matthews-Olsen complex. J. Phys. Chem. Lett. 2011, 2, 808-812.

(52) Kim, H. W.; Rhee, Y. M. Improving long time behavior of Poisson bracket mapping equation: A non-Hamiltonian approach. J. Chem. Phys. 2014, 140, 184106.

(53) Freedman, H.; Hanna, G. Mixed quantum-classical Liouville simulation of vibrational energy transfer in a model alpha-helix at 300 k. Chem. Phys. 2016, 477, 74-87.

(54) Bonella, S.; Coker, D. F. Semi-classical implementation of mapping Hamiltonian methods for general non-adiabatic problems. Chem. Phys. 2001, 268, 189-200.

(55) Bonella, S.; Coker, D. F. A semiclassical limit for the mapping hamiltonian approach to electronically nonadiabatic dynamics. J. Chem. Phys. 2001, 114, 7778-7789.

(56) Huo, P. F.; Coker, D. F. Consistent schemes for non-adiabatic dynamics derived from partial linearized density matrix propagation. J. Chem. Phys. 2012, 137, 22 a535.

(57) Huo, P.; III, T. F. M.; Coker, D. F. Communication: Predictive partial linearized path integral simulation of condensed phase electron transfer dynamics. J. Chem. Phys. 2013, 139, 151103.

(58) Provazza, J.; Segatta, F.; Garavelli, M.; Coker, D. F. Semiclassical path integral calculation of nonlinear optical spectroscopy. J. Chem. Theory Comput. 2018, 14, 856-866.

(59) Ananth, N.; Miller, T. F. Exact quantum statistics for electronically nonadiabatic systems using continuous path variables. J. Chem. Phys. 2010, 133, 234103.

(60) Tao, G. A multi-state trajectory method for non-adiabatic dynamics simulations. J. Chem. Phys. 2016, $144,094108$.

(61) Tao, G. H. Coherence-controlled nonadiabatic dynamics via state-space decomposition: A consistent way to incorporate Ehrenfest and Born-Oppenheimer-like treatments of nuclear motion. J. Phys. Chem. Lett. 2016, 7, 4335-4339.

(62) Liao, J.-L.; Voth, G. A. A centroid molecular dynamics approach for nonadiabatic dynamical processes in condensed phases: The spin-boson case. J. Phys. Chem. B 2002, 106, 8449-8455. 
(63) Ananth, N. Mapping variable ring polymer molecular dynamics: A path-integral based method for nonadiabatic processes. J. Chem. Phys. 2013, 139, 124102.

(64) Pierre, S.; Duke, J. R.; Hele, T. J. H.; Ananth, N. A mapping variable ring polymer molecular dynamics study of condensed phase proton-coupled electron transfer. J. Chem. Phys. 2017, 147, 234103.

(65) Chowdhury, S. N.; Huo, P. Coherent state mapping ring polymer molecular dynamics for nonadiabatic quantum propagations. J. Chem. Phys. 2017, 147, 214109.

(66) Richardson, J. O.; Meyer, P.; Pleinert, M. O.; Thoss, M. An analysis of nonadiabatic ring-polymer molecular dynamics and its application to vibronic spectra. Chem. Phys. 2017, 482, 124-134.

(67) Richardson, J. O.; Thoss, M. Communication: Nonadiabatic ring-polymer molecular dynamics. $J$. Chem. Phys. 2013, 139, 031102.

(68) Ranya, S.; Ananth, N. Multistate ring polymer instantons and nonadiabatic reaction rates. J. Chem. Phys. 2020, 152, 114112.

(69) Chowdhury, S. N.; Huo, P. F. State dependent ring polymer molecular dynamics for investigating excited nonadiabatic dynamics. J. Chem. Phys. 2019, 150, 244102.

(70) Müller, U.; Stock, G. Flow of zero-point energy and exploration of phase space in classical simulations of quantum relaxation dynamics. II. Application to nonadiabatic processes. J. Chem. Phys. 1999, 111, 77-88.

(71) Stock, G.; Müller, U. Flow of zero-point energy and exploration of phase space in classical simulations of quantum relaxation dynamics. J. Chem. Phys. 1999, 111, 65-76.

(72) Golosov, A. A.; Reichman, D. R. Classical mapping approaches for nonadiabatic dynamics: Short time analysis. J. Chem. Phys. 2001, 114, 1065-1074.

(73) Cotton, S. J.; Igumenshchev, K.; Miller, W. H. Symmetrical windowing for quantum states in quasi-classical trajectory simulations: Application to electron transfer. J. Chem. Phys. 2014, 141, 084104.

(74) Miller, W. H.; Cotton, S. J. Communication: Note on detailed balance in symmetrical quasi- 
classical models for electronically non-adiabatic dynamics. J. Chem. Phys. 2015, 142, 131103.

(75) Cotton, S. J.; Liang, R. B.; Miller, W. H. On the adiabatic representation of Meyer-Miller electronic-nuclear dynamics. J. Chem. Phys. 2017, 147, 064112.

(76) Jain, A.; Subotnik, J. E. Vibrational energy relaxation: A benchmark for mixed quantum-classical methods. J. Phys. Chem. A 2018, 122, 16-27.

(77) Bellonzi, N.; Jain, A.; Subotnik, J. E. An assessment of mean-field mixed semiclassical approaches: Equilibrium populations and algorithm stability. J. Chem. Phys. 2016, 144, 154110.

(78) Li, T. E.; Nitzan, A.; Sukharev, M.; Martínez, T.; Chen, H. T.; Subotnik, J. E. Mixed quantumclassical electrodynamics: Understanding spontaneous decay and zero-point energy. Phys. Rev. A 2018, 97, 032105.

(79) Kananenka, A. A.; Hsieh, C.-Y.; Cao, J.; Geva, E. Nonadiabatic dynamics via the symmetrical quasi-classical method in the presence of anharmonicity. J. Phys. Chem. Lett. 2017, 9, 319-326.

(80) Cotton, S. J.; Miller, W. H. A new symmetrical quasi-classical model for electronically nonadiabatic processes: Application to the case of weak non-adiabatic coupling. J. Chem. Phys. 2016, $145,144108$.

(81) Cotton, S. J.; Miller, W. H. The symmetrical quasi-classical model for electronically non-adiabatic processes applied to energy transfer dynamics in site-exciton models of light-harvesting complexes. J. Chem. Theory Comput. 2016, 12, 983-991.

(82) Cotton, S. J.; Miller, W. H. Symmetrical windowing for quantum states in quasi-classical trajectory simulations: Application to electronically non-adiabatic processes. J. Chem. Phys. 2013, $139,234112$.

(83) Tao, G. Understanding electronically non-adiabatic relaxation dynamics in singlet fission. J. Chem. Theory Comput. 2015, 11, 28-36.

(84) Tao, G. Electronically nonadiabatic dynamics in singlet fission: A quasi-classical trajectory simulation. J. Phys. Chem. C 2014, 118, 17299-17305.

(85) Tao, G. Bath effect in singlet fission dynamics. J. Phys. Chem. C 2014, 118, 27258-27264. 
(86) Liang, R.; Cotton, S. J.; Binder, R.; Hegger, R.; Burghardt, I.; Miller, W. H. The symmetrical quasi-classical approach to electronically nonadiabatic dynamics applied to ultrafast exciton migration processes in semiconducting polymers. J. Chem. Phys. 2018, 149, 044101.

(87) Xie, Y.; Zheng, J.; Lan, Z. Performance evaluation of the symmetrical quasi-classical dynamics method based on Meyer-Miller mapping Hamiltonian in the treatment of site-exciton models. $J$. Chem. Phys. 2018, 149, 174105.

(88) Sandoval_C., J. S.; Mandal, A.; Huo, P. Symmetric quasi-classical dynamics with quasi-diabatic propagation scheme. J. Chem. Phys. 2018, 149, 044115.

(89) Provazza, J.; Coker, D. F. Communication: Symmetrical quasi-classical analysis of linear optical spectroscopy. J. Chem. Phys. 2018, 148, 181102.

(90) Zheng, J.; Peng, J. W.; Xie, Y.; Long, Y. Z.; Ning, X.; Lan, Z. G. Study of the exciton dynamics in perylene bisimide (PBI) aggregates with symmetrical quasiclassical dynamics based on the Meyer-Miller mapping hamiltonian. Phys. Chem. Chem. Phys. 2020, 22, 18192-18204.

(91) Cotton, S. J.; Miller, W. H. A symmetrical quasi-classical windowing model for the molecular dynamics treatment of non-adiabatic processes involving many electronic states. J. Chem. Phys. 2019, $150,104101$.

(92) Cotton, S. J.; Miller, W. H. Trajectory-adjusted electronic zero point energy in classical MeyerMiller vibronic dynamics: Symmetrical quasiclassical application to photodissociation. J. Chem. Phys. 2019, 150, 194110.

(93) Zheng, J.; Xie, Y.; Jiang, S. s.; Long, Y. z.; Ning, X.; Lan, Z. G. Ultrafast electron transfer with symmetrical quasi-classical dynamics based on mapping hamiltonian and quantum dynamics based on ml-mctdh. Chin. J. Chem. Phys. 2017, 30, 800-810.

(94) Mandal, A.; Sandoval C, J. S.; Shakib, F. A.; Huo, P. Quasi-diabatic propagation scheme for direct simulation of proton-coupled electron transfer reaction. J. Phys. Chem. A 2019, 123, 2470-2482.

(95) Mandal, A.; Yamijala, S.; Huo, P. F. Quasi-diabatic representation for nonadiabatic dynamics propagation. J. Chem. Theory Comput. 2018, 14, 1828-1840. 
(96) Mandal, A.; Shakib, F. A.; Huo, P. Investigating photoinduced proton coupled electron transfer reaction using quasi diabatic dynamics propagation. J. Chem. Phys. 2018, 148, 244102.

(97) Zhou, W.; Mandal, A.; Huo, P. Quasi-diabatic scheme for nonadiabatic on-the-fly simulations. $J$. Phys. Chem. Lett. 2019, 10, 7062-7070.

(98) Mead, C. A.; Truhlar, D. G. Conditions for the Definition of a Strictly Diabatic Electronic Basis for Molecular-Systems. J. Chem. Phys. 1982, 77, 6090-6098.

(99) McLachlan, A. The wave functions of electronically degenerate states. Mol. Phys. 1961, 4, 417423.

(100) Pacher, T.; Mead, C. A.; Cederbaum, L. S.; Koppel, H. Gauge-theory and quasidiabatic states in molecular physics. J. Chem. Phys. 1989, 91, 7057-7062.

(101) Baer, M. Adiabatic and diabatic representations for atom-molecule collisions: Treatment of the collinear arrangement. Chem. Phys. Lett. 1975, 35, 112-118.

(102) Ananth, N.; Venkataraman, C.; Miller, W. H. Semiclassical description of electronically nonadiabatic dynamics via the initial value representation. J. Chem. Phys. 2007, 127, 084114.

(103) Coker, D. F.; Bonella, S., Linearized nonadiabatic dynamics in the adiabatic representation. In Springer Ser Chem Ph, 2007; pp 321-340.

(104) Hsieh, C.-Y.; Schofield, J.; Kapral, R. Forward-backward solution of quantum-classical Liouville equation in the adiabatic mapping basis. Mol. Phys. 2013, 111, 3546-3554.

(105) Tang, D. D.; Fang, W. H.; Shen, L.; Cui, G. L. Combining Meyer-Miller hamiltonian with electronic structure methods for on-the-fly nonadiabatic dynamics simulations: Implementation and application. Phys. Chem. Chem. Phys. 2019, 21, 17109-17117.

(106) Du, L. K.; Lan, Z. G. An on-the-fly surface-hopping program JADE for nonadiabatic molecular dynamics of polyatomic systems: Implementation and applications. J. Chem. Theory Comput. 2015, 11, 1360-1374.

(107) Hu, D.; Liu, Y. F.; Sobolewski, A. L.; Lan, Z. Nonadiabatic dynamics simulation of keto isocytosine: a comparison of dynamical performance of different electronic-structure methods. 
Phys. Chem. Chem. Phys. 2017, 19, 19168-19177.

(108) Fabiano, E.; Keal, T. W.; Thiel, W. Implementation of surface hopping molecular dynamics using semiempirical methods. Chem. Phys. 2008, 349, 334-347.

(109) Hollas, D.; Šištík, L.; Hohenstein, E. G.; Martínez, T. J.; Slavíček, P. Nonadiabatic ab initio molecular dynamics with the floating occupation molecular orbital-complete active space configuration interaction method. J. Chem. Theory Comput. 2018, 14, 339-350.

(110) Barbatti, M.; Aquino, A. J. A.; Lischka, H. Ultrafast two-step process in the non- adiabatic relaxation of the $\mathrm{CH} 2 \mathrm{NH} 2+$ molecule. Mol. Phys. 2006, 104, 1053-1060.

(111) Barbatti, M.; Granucci, G.; Persico, M.; Ruckenbauer, M.; Vazdar, M.; Eckert-Maksić, M.; Lischka, H. The on-the-fly surface-hopping program system NEWTON-X: Application to ab initio simulation of the nonadiabatic photodynamics of benchmark systems. J. Photoch. Photobio. A 2007, 190, 228-240.

(112) Du, L. K.; Lan, Z. G. Correction to "an on-the-fly surface-hopping program JADE for nonadiabatic molecular dynamics of polyatomic systems: Implementation and applications". $J$. Chem. Theory Comput. 2015, 11, 4522-4523.

(113) Kunisada, T.; Ushiyama, H.; Yamashita, K. A new implementation of ab initio Ehrenfest dynamics using electronic configuration basis: Exact formulation with molecular orbital connection and effective propagation scheme with locally quasi-diabatic representation. Int. J. Quantum Chem. 2016, 116, 1205-1213.

(114) Kunisada, T.; Ushiyama, H.; Yamashita, K. Electron wavepacket approaches to non-adiabatic transition processes in the internal rotational motion of $\mathrm{H} 2 \mathrm{CNH} 2+-$ charge oscillation due to electronic coherence. Chem. Phys. Lett. 2015, 635, 345-349.

(115) Fischer, M.; Handt, J.; Schmidt, R. Nonadiabatic quantum molecular dynamics with hopping. III. Photoinduced excitation and relaxation of organic molecules. Phys. Rev. A 2014, 90, 012527.

(116) West, A. C.; Barbatti, M.; Lischka, H.; Windus, T. L. Nonadiabatic dynamics study of methaniminium with ORMAS: Challenges of incomplete active spaces in dynamics simulations. 
Comput. Theor. Chem. 2014, 1040, 158-166.

(117) Zimmermann, T.; Vanicek, J. Evaluation of the importance of spin-orbit couplings in the nonadiabatic quantum dynamics with quantum fidelity and with its efficient "on-the-fly" ab initio semiclassical approximation. J. Chem. Phys. 2012, 137, 22A516.

(118) Tavernelli, I.; Tapavicza, E.; Rothlisberger, U. Non-adiabatic dynamics using time-dependent density functional theory: Assessing the coupling strengths. J. Mol. Struct. 2009, 914, $22-29$.

(119) Fabiano, E.; Groenhof, G.; Thiel, W. Approximate switching algorithms for trajectory surface hopping. Chem. Phys. 2008, 351, 111-116.

(120) Gaenko, A.; DeFusco, A.; Varganov, S. A.; Martínez, T. J.; Gordon, M. S. Interfacing the ab initio multiple spawning method with electronic structure methods in GAMESS: Photo-decay of trans-azomethane. J. Phys. Chem. A 2014, 118, 10902-10908.

(121) Sellner, B.; Ruckenbauer, M.; Stambolic, I.; Barbatti, M.; Aquino, A. J.; Lischka, H. Photodynamics of azomethane: A nonadiabatic surface-hopping study. J. Phys. Chem. A 2010, $114,8778-8785$.

(122) Frisch, M. J.; Trucks, G. W.; Schlegel, H. B.; Scuseria, G. E.; Robb, M. A.; Cheeseman, J. R.; Scalmani, G.; Barone, V.; Petersson, G. A.; Nakatsuji, H.; Li, X.; Caricato, M.; Marenich, A. V.; Bloino, J.; Janesko, B. G.; Gomperts, R.; Mennucci, B.; Hratchian, H. P.; Ortiz, J. V.; Izmaylov, A. F.; Sonnenberg, J. L.; Williams; Ding, F.; Lipparini, F.; Egidi, F.; Goings, J.; Peng, B.; Petrone, A.; Henderson, T.; Ranasinghe, D.; Zakrzewski, V. G.; Gao, J.; Rega, N.; Zheng, G.; Liang, W.; Hada, M.; Ehara, M.; Toyota, K.; Fukuda, R.; Hasegawa, J.; Ishida, M.; Nakajima, T.; Honda, Y.; Kitao, O.; Nakai, H.; Vreven, T.; Throssell, K.; Montgomery Jr., J. A.; Peralta, J. E.; Ogliaro, F.; Bearpark, M. J.; Heyd, J. J.; Brothers, E. N.; Kudin, K. N.; Staroverov, V. N.; Keith, T. A.; Kobayashi, R.; Normand, J.; Raghavachari, K.; Rendell, A. P.; Burant, J. C.; Iyengar, S. S.; Tomasi, J.; Cossi, M.; Millam, J. M.; Klene, M.; Adamo, C.; Cammi, R.; Ochterski, J. W.; Martin, R. L.; Morokuma, K.; Farkas, O.; Foresman, J. B.; Fox, D. J. Gaussian 16 Rev. C.01, Wallingford, CT, 2016. 
(123) Barbatti, M. Nonadiabatic dynamics with trajectory surface hopping method. Wiley Interdiscip. Rev.: Comput. Mol. Sci. 2011, 1, 620-633.

(124) Werner, H. J.; Knowles, P. J. A second order multiconfiguration SCF procedure with optimum convergence. J. Chem. Phys. 1985, 82, 5053-5063.

(125) Knowles, P. J.; Werner, H.-J. An efficient second-order MCSCF method for long configuration expansions. Chem. Phys. Lett. 1985, 115, 259-267.

(126) Werner, H. J.; Knowles, P. J.; Knizia, G.; Manby, F. R.; Schutz, M. Molpro, version 2012.1, a package of ab initio programs, 2012.

(127) Barbatti, M.; Ruckenbauer, M.; Plasser, F.; Pittner, J.; Granucci, G.; Persico, M.; Lischka, H. Newton-X: A surface-hopping program for nonadiabatic molecular dynamics. Wiley Interdiscip. Rev.: Comput. Mol. Sci. 2014, 4, 26-33.

(128) Tapavicza, E.; Tavernelli, I.; Rothlisberger, U. Trajectory surface hopping within linear response time-dependent density-functional theory. Phys. Rev. Lett. 2007, 98, 023001.

(129) Diau, E. W.; Zewail, A. H. Femtochemistry of trans-azomethane: a combined experimental and theoretical study. Chemphyschem 2003, 4, 445-56.

(130) Barbatti, M.; Lan, Z.; Crespo-Otero, R.; Szymczak, J. J.; Lischka, H.; Thiel, W. Critical appraisal of excited state nonadiabatic dynamics simulations of 9H-adeninea). J. Chem. Phys. 2012, 137, 22A503.

(131) Landry, B. R.; Subotnik, J. E. How to recover Marcus theory with fewest switches surface hopping: Add just a touch of decoherence. J. Chem. Phys. 2012, 137, 22 A513. 
Supporting information for

\section{On-the-fly Symmetrical Quasi-classical Dynamics with Meyer-Miller Mapping Hamiltonian for the Treatment of Nonadiabatic Dynamics at Conical Intersections}

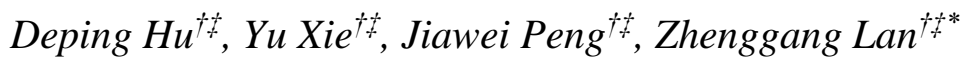

${ }^{\dagger}$ SCNU Environmental Research Institute, Guangdong Provincial Key Laboratory of Chemical Pollution and Environmental Safety \& MOE Key Laboratory of Environmental Theoretical Chemistry, South China Normal University, Guangzhou 510006, China

${ }^{t}$ School of Environment, South China Normal University, Guangzhou 510006, China

Email: zhenggang.lan@m.scnu.edu.cn; zhenggang.lan@gmail.com 

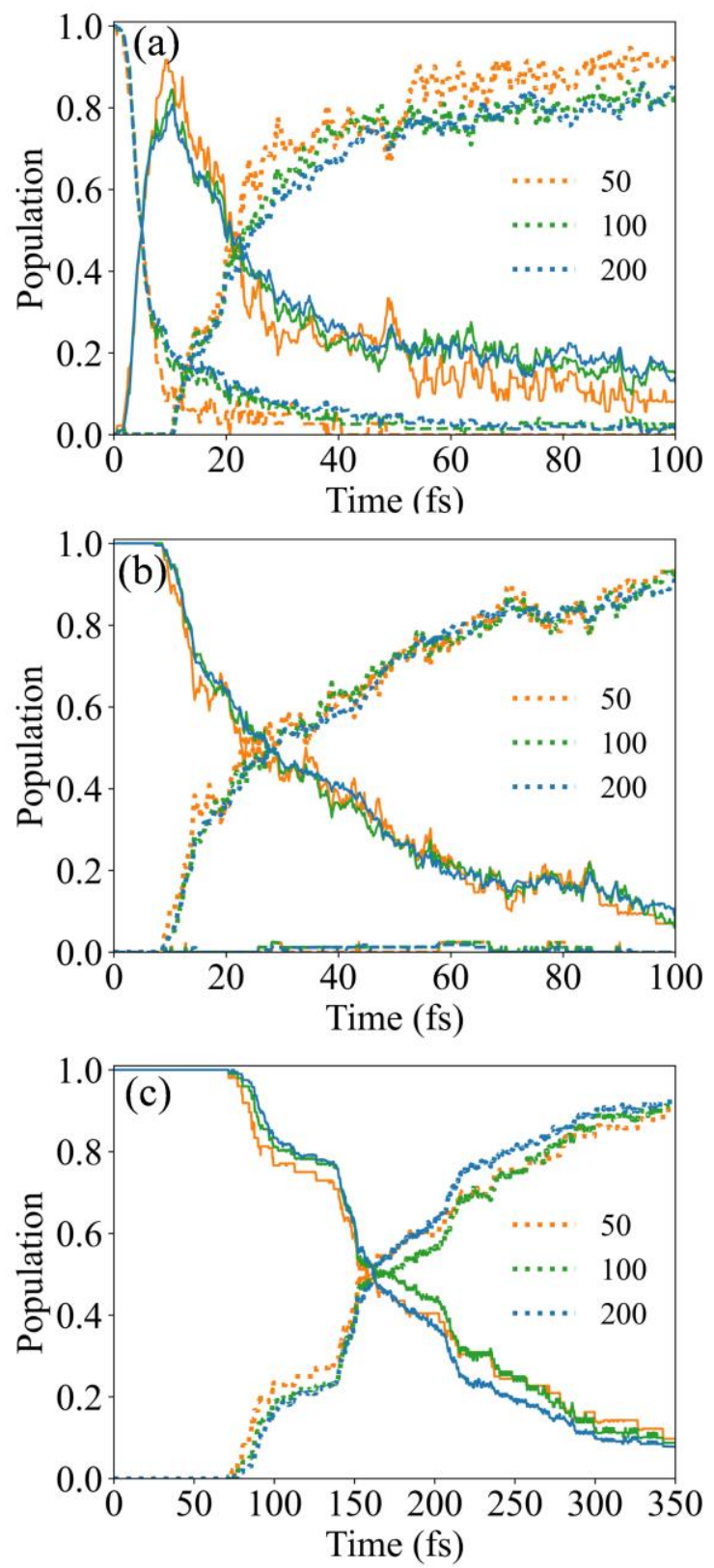

Figure $\mathrm{S} 1$. The time-dependent electronic populations of $\mathrm{S}_{0}$ (dotted line), $\mathrm{S}_{1}$ (solid line) and $\mathrm{S}_{2}$ (dashed line) states of $\gamma$-adjusted SQC/MM dynamics for (a) $\mathrm{CH}_{2} \mathrm{NH}_{2}{ }^{+}$starting from $\mathrm{S}_{2}$, (b) $\mathrm{CH}_{2} \mathrm{NH}_{2}{ }^{+}$starting $\mathrm{S}_{1}$ and (c) azomethane starting from $\mathrm{S}_{1}$. The number of trajectories is given in each subfigure. In the main text, 200 trajectories were used to discuss the results. 

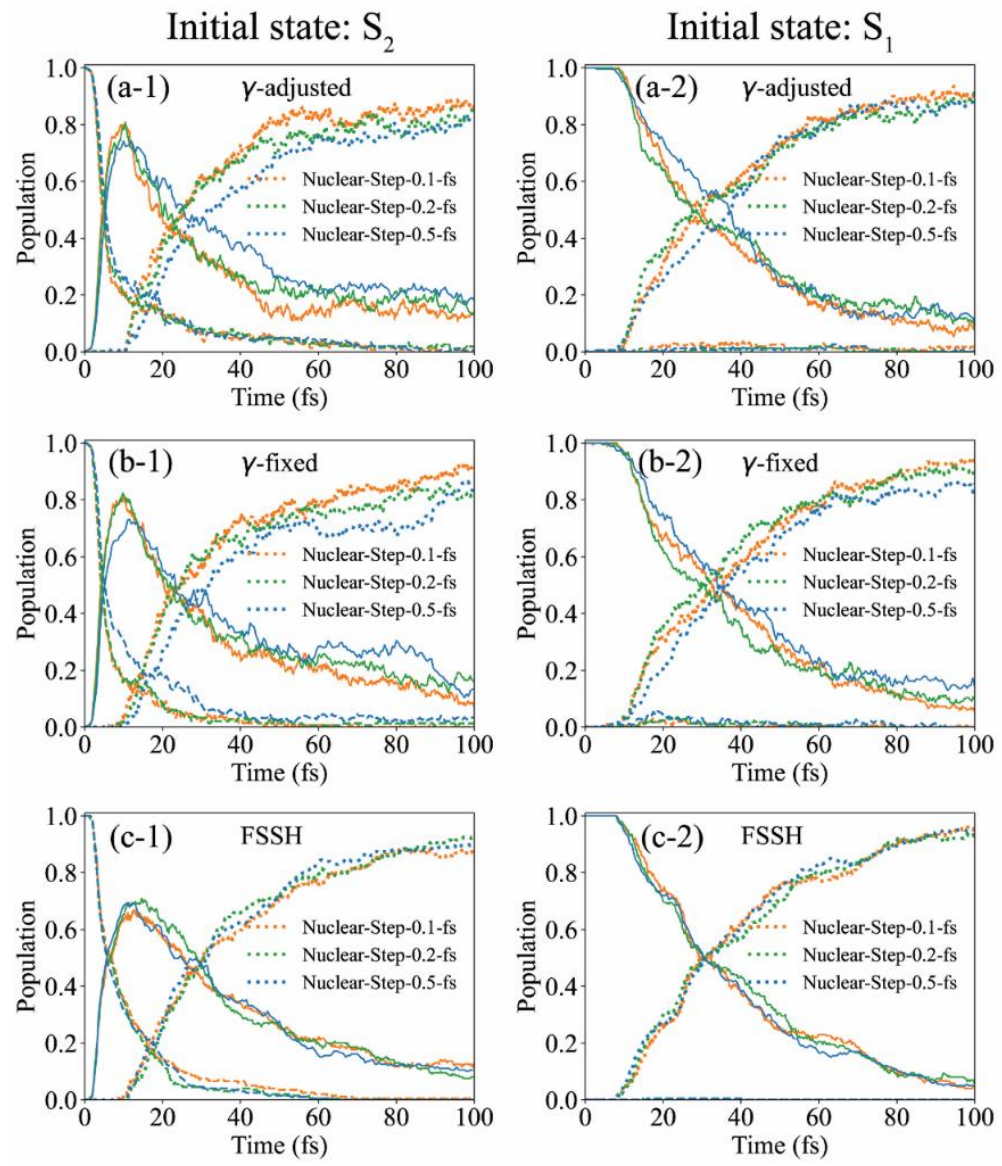

Figure S2. The time-dependent electronic populations of $\mathrm{S}_{0}$ (dotted line), $\mathrm{S}_{1}$ (solid line) and $\mathrm{S}_{2}$ (dashed line) for (a) $\gamma$-adjusted SQC/MM, (b) $\gamma$-fixed SQC/MM and (c) FSSH dynamics for $\mathrm{CH}_{2} \mathrm{NH}_{2}{ }^{+}$starting from (1) $\mathrm{S}_{2}$ and (2) $\mathrm{S}_{1}$. The numbers in the legends represent the nuclear time steps used in the dynamics. For each step of nuclear motion, 100 steps of electronic motion were performed. 200 trajectories were used for all dynamics.

For SQC/MM dynamics, the time-dependent electronic populations with nuclear time steps of 0.1 fs and 0.2 fs are very close, while a few different results are given with 0.5 fs. For FSSH dynamics, the time-dependent electronic populations change very little from time step of 0.1 fs to $0.5 \mathrm{fs}$. Therefore, we concluded that the nuclear time steps of $0.2 \mathrm{fs}$ and $0.5 \mathrm{fs}$ are enough for SQC/MM and FSSH dynamics, respectively. 
From $\mathrm{S}_{2}$
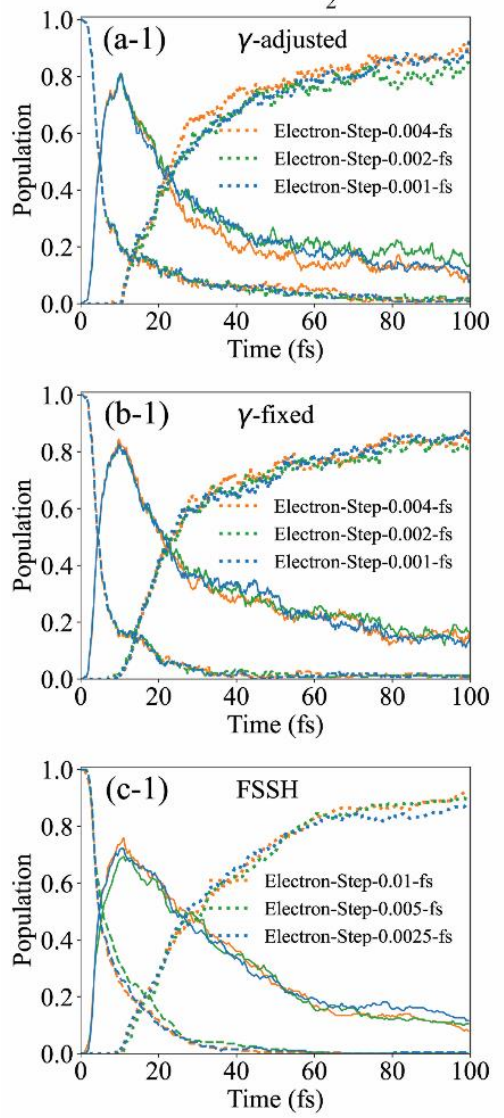

From $\mathrm{S}_{1}$
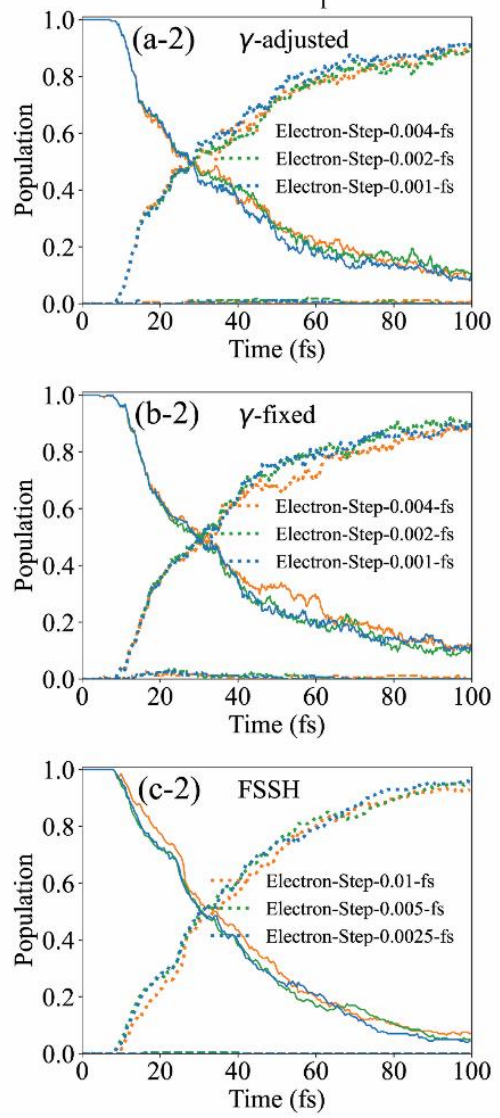

Figure S3. The time-dependent electronic populations of $\mathrm{S}_{0}$ (dotted line), $\mathrm{S}_{1}$ (solid line) and $\mathrm{S}_{2}$ (dashed line) for (a) $\gamma$-adjusted SQC/MM, (b) $\gamma$-fixed SQC/MM and (c) FSSH dynamics for $\mathrm{CH}_{2} \mathrm{NH}_{2}{ }^{+}$starting from (1) $\mathrm{S}_{2}$ and (2) $\mathrm{S}_{1}$. The numbers in the legends represent the electronic time steps used in the dynamics. Nuclear time steps of $0.2 \mathrm{fs}$ and $0.5 \mathrm{fs}$ were used for SQC/MM and FSSH dynamics, respectively. 200 trajectories were used for all dynamics.

For SQC/MM dynamics, the time-dependent electronic populations with electronic time steps of 0.004, 0.002 and 0.001 fs are all very close. For FSSH dynamics, the time-dependent electronic populations with electronic time steps of $0.005 \mathrm{fs}$ and $0.0025 \mathrm{fs}$ are very close, while a few different results are given with $0.01 \mathrm{fs}$. Therefore, we concluded that the electronic time steps of $0.004 \mathrm{fs}$ (1/50 of corresponding nuclear time step of $0.2 \mathrm{fs})$ and $0.005 \mathrm{fs}(1 / 100$ of corresponding nuclear time step of $0.5 \mathrm{fs}$ ) are enough for SQC/MM and FSSH dynamics, respectively. Since the propagation of the electronic motion is not very time-consuming in the on-the-fly dynamics, we used 1/100 of nuclear time steps to obtain the electronic time step for all dynamics. 
Initial state: $\mathrm{S}_{2}$
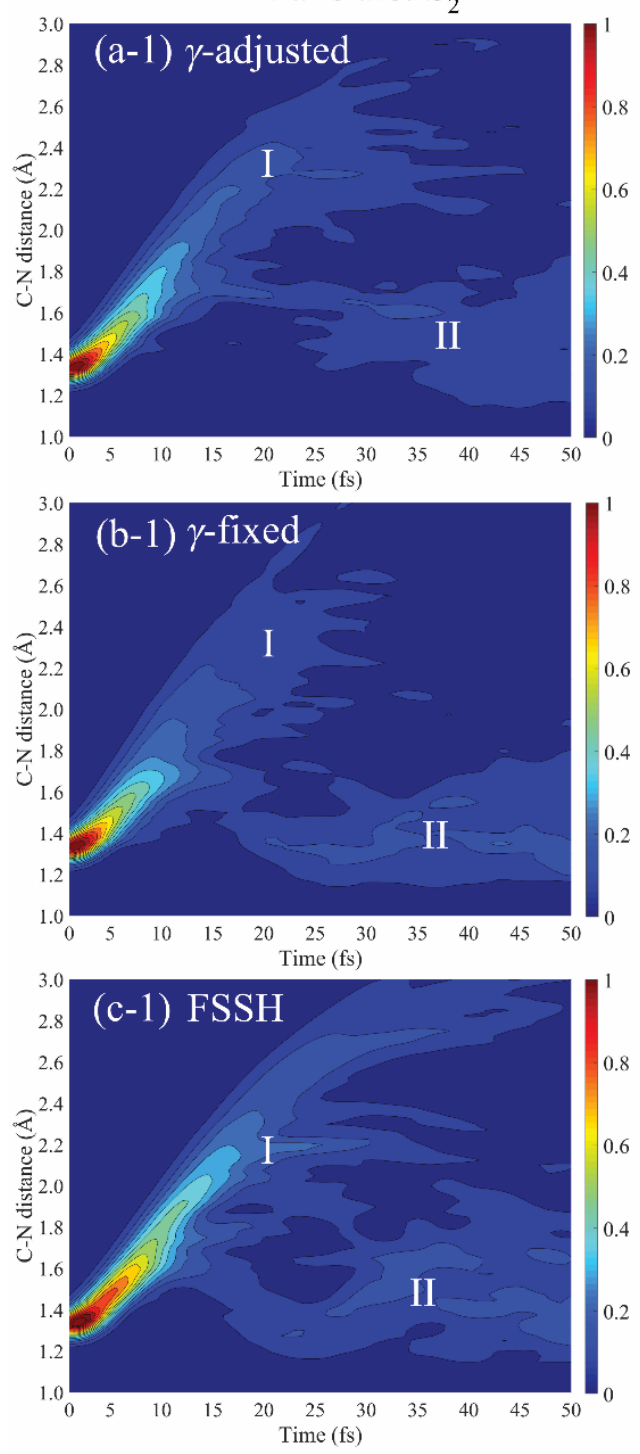

Initial state: $\mathrm{S}_{1}$
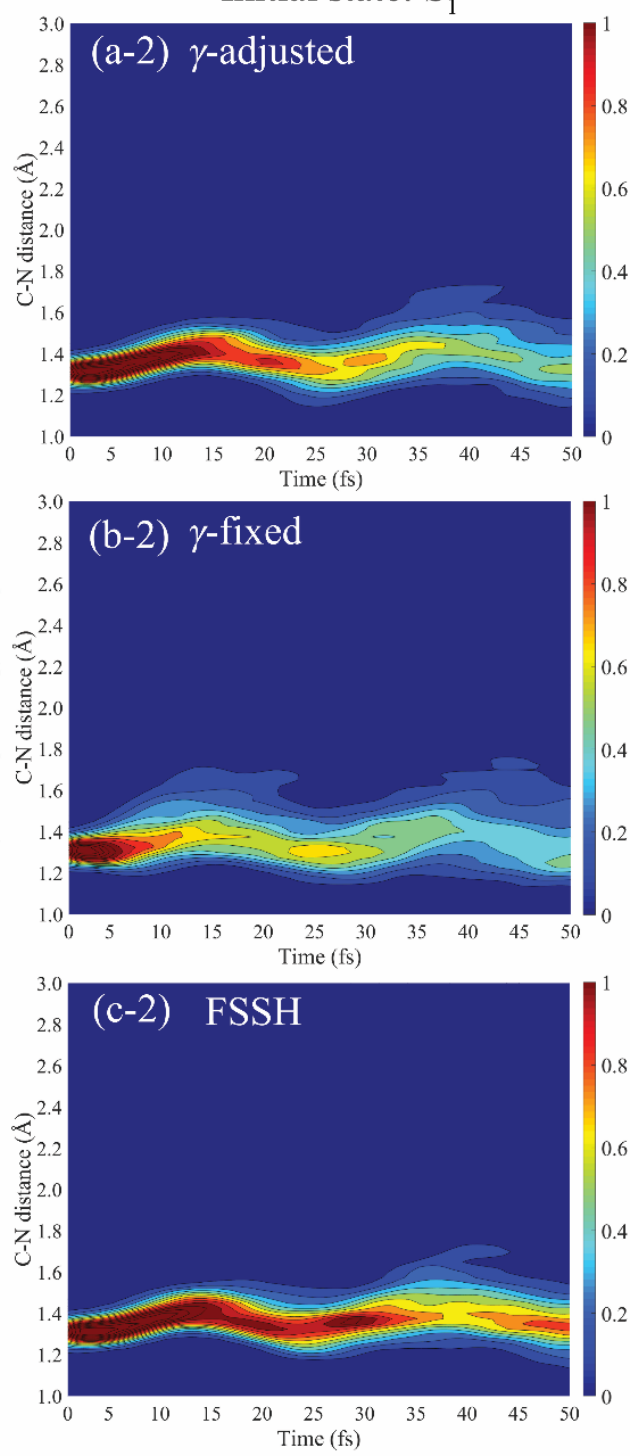

Figure S4. The time-dependent $\mathrm{CN}$ distance distributions of $\mathrm{CH}_{2} \mathrm{NH}_{2}{ }^{+}$in the (a) $\gamma$-adjusted, (b) $\gamma$ fixed SQC/MM and (c) FSSH dynamics. The initial states are (1) $\mathrm{S}_{2}$ and (2) $\mathrm{S}_{1}$, respectively. Only the geometries in the windows are included in SQC/MM dynamics. 200 trajectories were used for all dynamics. 

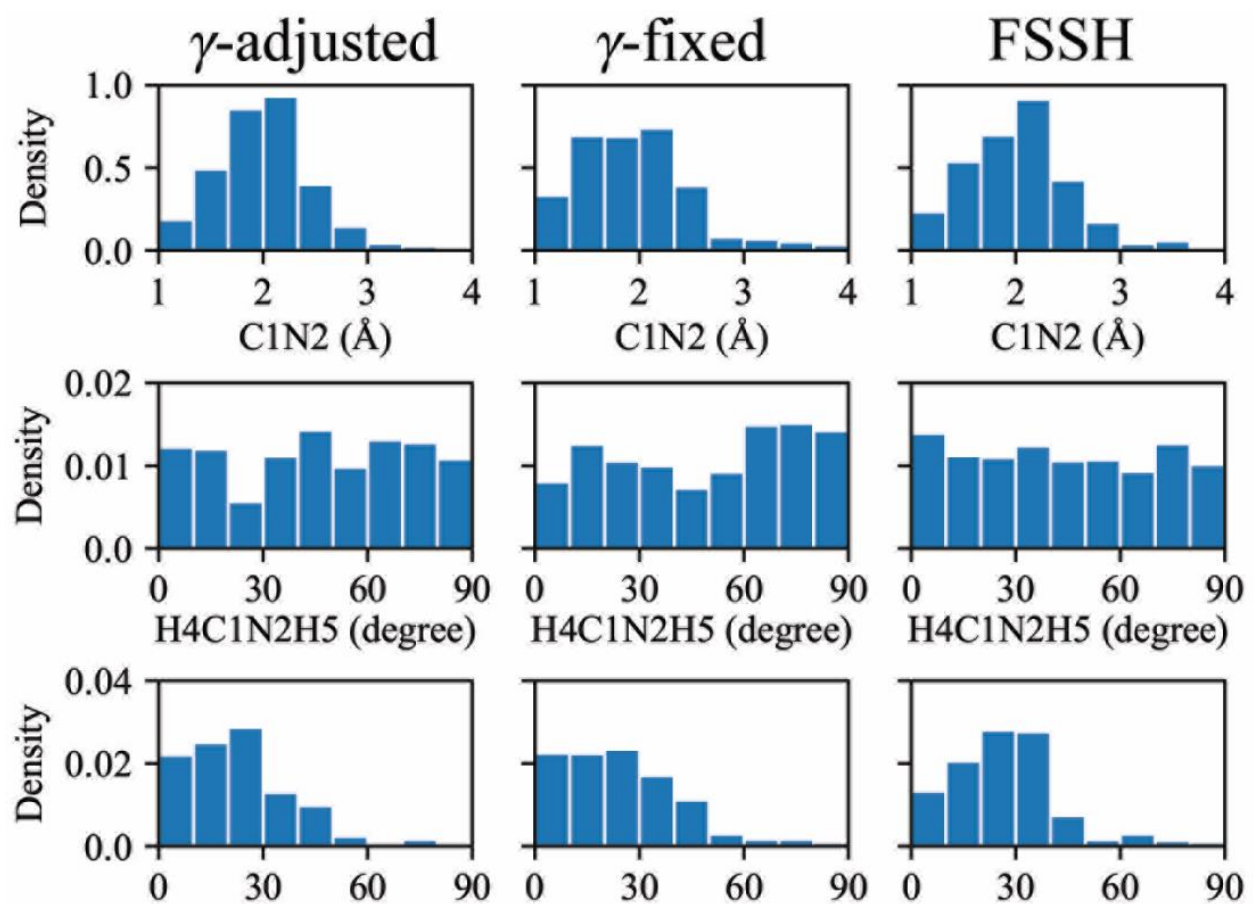

$\mathrm{H} 4 \mathrm{H} 3 \mathrm{~N} 2 \mathrm{C} 1$ (degree)
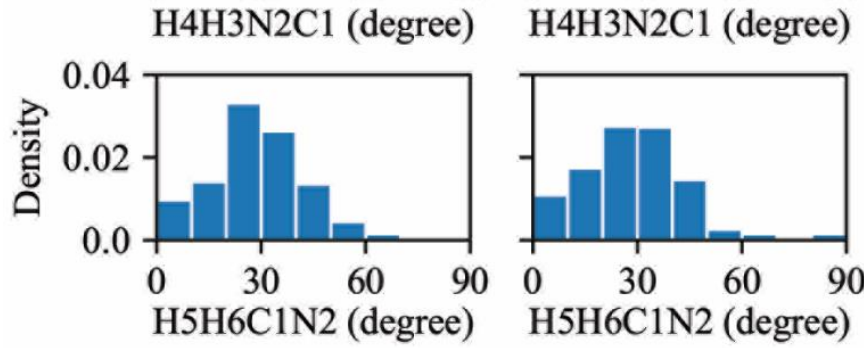

$\mathrm{H} 4 \mathrm{H} 3 \mathrm{~N} 2 \mathrm{C} 1$ (degree)
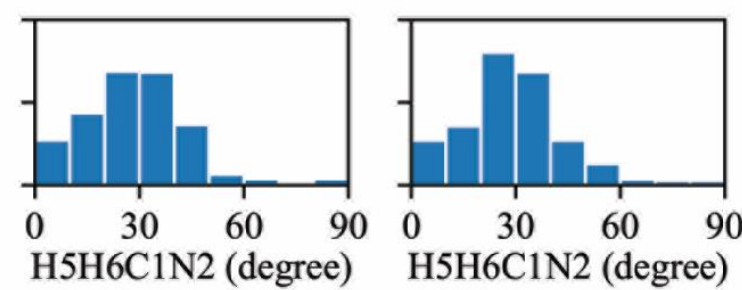

Figure S5. Normalized distributions of key degrees of freedom: bond length C1N2 (CN stretching), dihedral angle $\mathrm{H} 4 \mathrm{C} 1 \mathrm{~N} 2 \mathrm{H} 5$ ( $\mathrm{CN}$ torsion angle), dihedral angle $\mathrm{H} 4 \mathrm{H} 3 \mathrm{~N} 2 \mathrm{C} 1$ (pyramidalization at $\mathrm{C}$ atom) and dihedral angle $\mathrm{H} 5 \mathrm{H} 6 \mathrm{C} 1 \mathrm{~N} 2$ (pyramidalization at $\mathrm{N}$ atom) of the $\mathrm{CH}_{2} \mathrm{NH}_{2}{ }^{+}$molecule at five $S_{1} / S_{0}$ minimum-energy-gap structures in $\gamma$-adjusted SQC/MM, $\gamma$-fixed SQC/MM and FSSH dynamics starting from $\mathbf{S}_{2}$. For all dynamics approaches, we do not consider the trajectory that does not return back $\mathrm{S}_{0}$. 

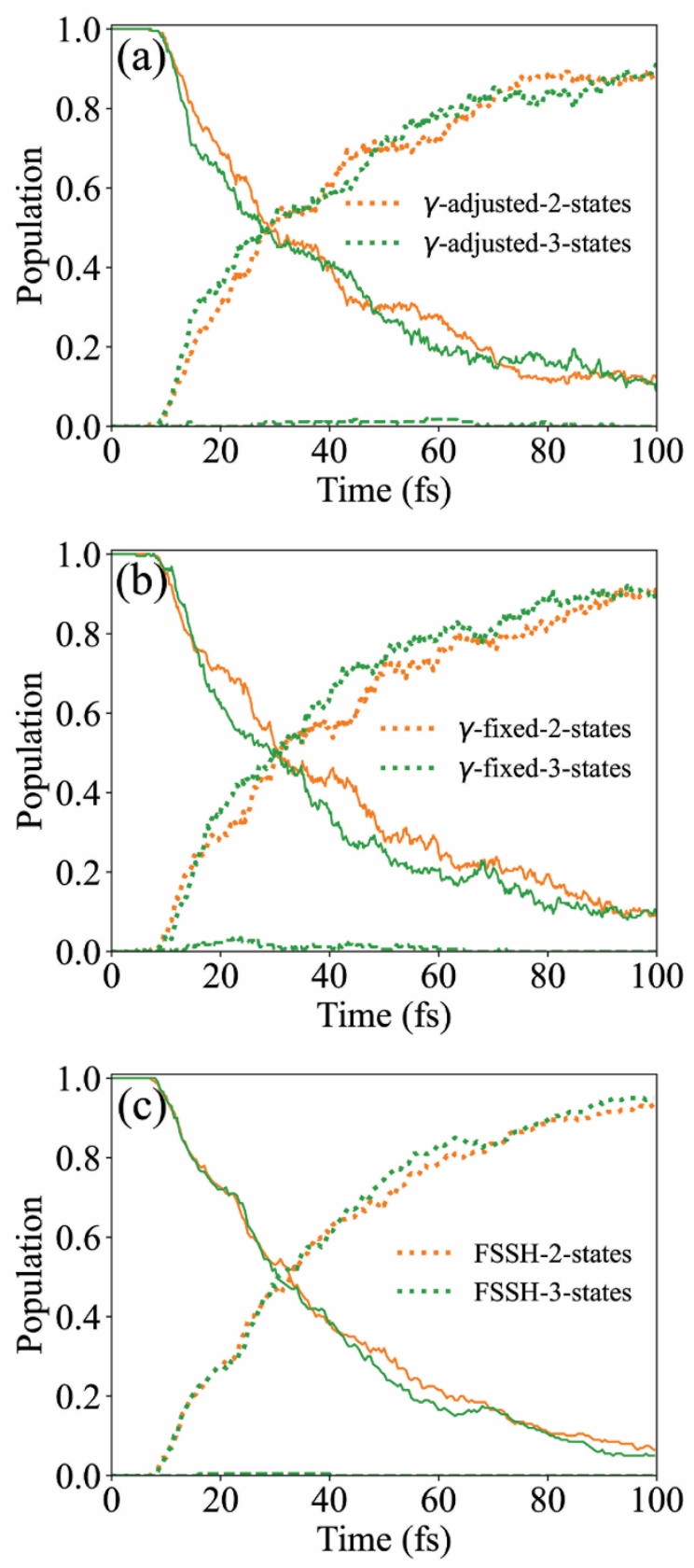

Figure S6. The time-dependent electronic populations of $\mathrm{S}_{0}$ (dotted line), $\mathrm{S}_{1}$ (solid line) and $\mathrm{S}_{2}$ (dashed line) states of (a) $\gamma$-adjusted SQC/MM, (b) $\gamma$-fixed SQC/MM and (c) FSSH dynamics for $\mathrm{CH}_{2} \mathrm{NH}_{2}{ }^{+}$molecule starting from $\mathrm{S}_{1}$. The number of states involved in the dynamics is given in each subfigure. 200 trajectories were used for all dynamics. 

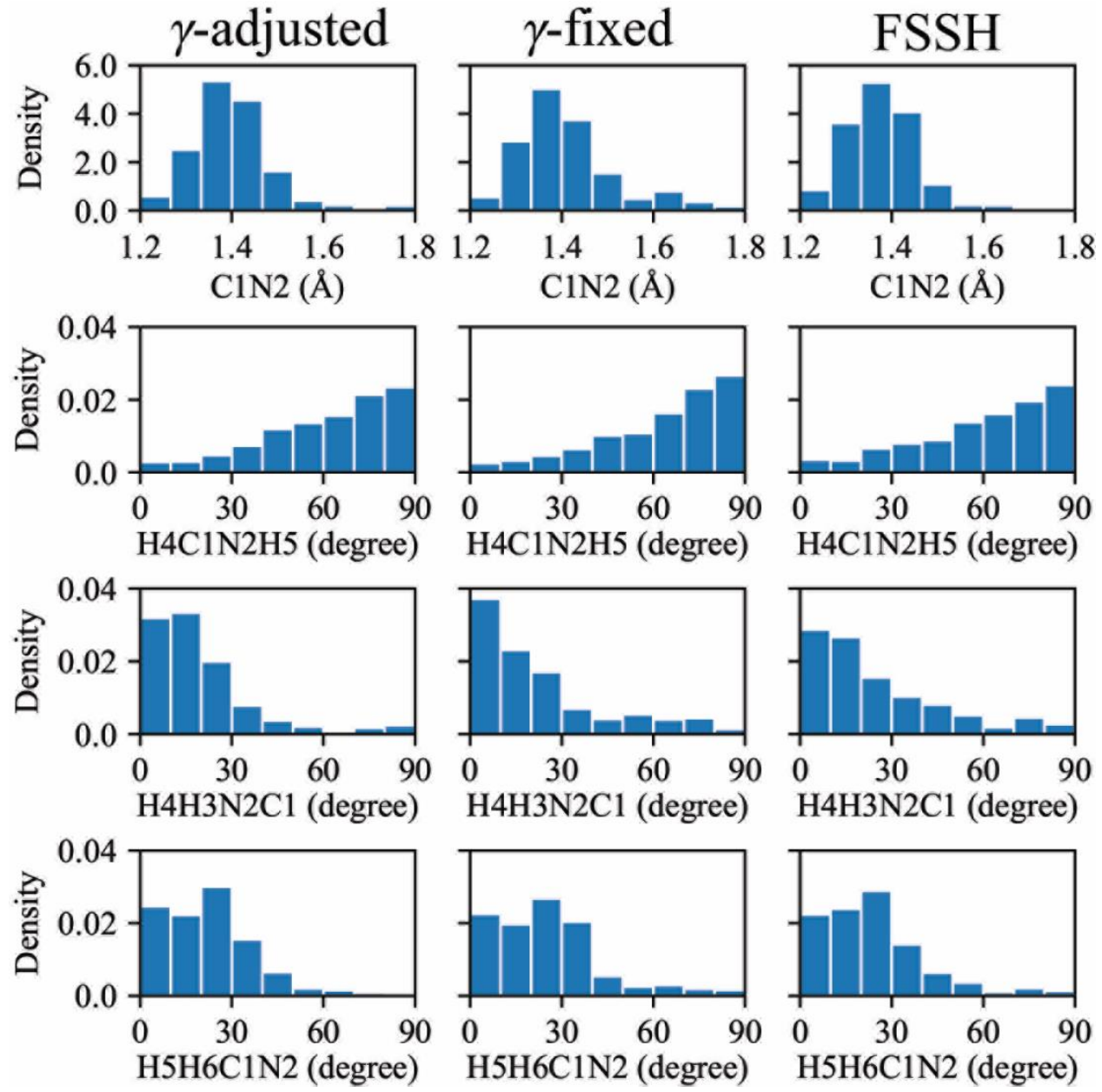

Figure S7. Normalized distributions of key degrees of freedom: bond length C1N2 (CN stretching), dihedral angle $\mathrm{H} 4 \mathrm{C} 1 \mathrm{~N} 2 \mathrm{H} 5$ (CN torsion angle), dihedral angle $\mathrm{H} 4 \mathrm{H} 3 \mathrm{~N} 2 \mathrm{C} 1$ (pyramidalization at $\mathrm{C}$ atom) and dihedral angle $\mathrm{H} 5 \mathrm{H} 6 \mathrm{C} 1 \mathrm{~N} 2$ (pyramidalization at $\mathrm{N}$ atom) of the $\mathrm{CH}_{2} \mathrm{NH}_{2}{ }^{+}$molecule at five $\mathrm{S}_{1} / \mathrm{S}_{0}$ minimum-energy-gap structures in $\gamma$-adjusted SQC/MM, $\gamma$-fixed SQC/MM and FSSH dynamics starting from $S_{1}$. For all dynamics approaches, we do not consider the trajectory that does not return back to $S_{0}$. 